\title{
Statistics of vertical backscatter profiles of cirrus clouds
}

\author{
P. Veglio and T. Maestri \\ Physics Department, Alma Mater Studiorum, University of Bologna, Bologna, Italy \\ Received: 23 July 2011 - Published in Atmos. Chem. Phys. Discuss.: 16 September 2011 \\ Revised: 29 November 2011 - Accepted: 8 December 2011 - Published: 20 December 2011
}

\begin{abstract}
A nearly global statistical analysis of vertical backscatter and extinction profiles of cirrus clouds collected by the CALIOP lidar, on-board of the Cloud-Aerosol Lidar and Infrared Pathfinder Satellite Observation, is presented.

Statistics on frequency of occurrence and distribution of bulk properties of cirrus clouds in general and, for the first time, of horizontally homogeneous (on a 5-km field of view) cirrus clouds only are provided. Annual and seasonal backscatter profiles (BSP) are computed for the horizontally homogeneous cirri. Differences found in the day/night cases and for midlatitudes and tropics are studied in terms of the mean physical parameters of the clouds from which they are derived.

The relationship between cloud physical parameters (optical depth, geometrical thickness and temperature) and the shape of the BSP is investigated. It is found that cloud geometrical thickness is the main parameter affecting the shape of the mean CALIOP BSP. Specifically, cirrus clouds with small geometrical thicknesses show a maximum in mean BSP curve located near cloud top. As the cloud geometrical thickness increases the BSP maximum shifts towards cloud base. Cloud optical depth and temperature have smaller effects on the shape of the CALIOP BSPs. In general a slight increase in the BSP maximum is observed as cloud temperature and optical depth increase.

In order to fit mean BSPs, as functions of geometrical thickness and position within the cloud layer, polynomial functions are provided. The impact on satellite radiative transfer simulations in the infrared spectrum when using either a constant ice-content (IWC) along the cloud vertical dimension or an IWC profile derived from the BSP fitting functions is evaluated. It is, in fact, demonstrated that, under realistic hypotheses, the mean BSP is linearly proportional to the IWC profile.
\end{abstract}

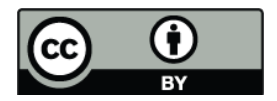

Correspondence to: P. Veglio

(paolo.veglio3@unibo.it)

\section{Introduction and motivations}

The persistence of high-level clouds, which normally cover more than $20 \%$ of the globe (Wylie et al., 1994), suggests that they play an important role in the Earth energy budget. At midlatitudes (ML) they exhibit an almost constant coverage and their coverage is about $40 \%$. At these latitudes ice clouds are generated both by large-scale ascent and atmospheric waves activity (Lynch, 2002). In the tropics, a maximum of occurrence has been observed near the equator $(\sim 70 \%)$ and a minimum one $(<10 \%)$ in the subtropical regions (Wylie et al., 2005). Deep convection is the engine that produces tropical ice clouds. In particular, thin cirrus clouds in the tropics are mainly formed by iced particles flowing out from the convective core of cumuli at cloud top.

The radiative impact of ice clouds is crucial for determining the energy balance of the Earth's atmosphere since their albedo and greenhouse effects are of the same order of magnitude (McFarquhar et al., 2000). In the upper troposphere, the energy balance in presence of ice clouds depends on several cloud parameters such as cloud top height and thickness, Ice-Water-Content (IWC), optical depth (OD) and ice particle size distribution (PSD) (Liou, 1986; Maestri and Rizzi, 2003).

The different cirrus cloud formation processes occurring at ML and tropics influence the distribution of mass, particle sizes and ice crystals habits within the clouds and possibly affect their radiative properties. Unfortunately, due to the very extreme conditions, in situ measurements of high cloud microphysical properties are technically difficult (McFarquhar and Heymsfield, 1996). In particular, field campaigns focused on the vertical characterization of ice clouds properties are few if compared to the large variability and occurrence of this type of clouds (Wendisch et al., 2005), and, thus, current databases do not appropriately reproduce the possible heterogeneity found in nature.

Despite the scarceness of data, the vertical distribution of physical and microphysical properties of clouds influences

Published by Copernicus Publications on behalf of the European Geosciences Union. 
their radiative features (Maestri et al., 2005) and interpretation of remote sensing data. At this purpose, Maestri and Holz (2009) show how the assumption on the vertical distribution of IWC might impact cloud properties retrievals (OD and particle effective dimension) from infrared satellite measurements. Chiriaco et al. (2006), while highlighting the potentialities of using combined visible $(532 \mathrm{~nm})$ and infrared $(10.6 \mu \mathrm{m})$ lidar observations for retrieving vertically resolved profiles of particle absorption efficiency, particle concentration and IWC, show the importance of determining the position of the maximum of absorption within a cloud layer and provide an example of the large variations in the retrievals of the particles effective sizes for different hypotheses on where maximum of absorption takes place. Along the same line of research, Zhang et al. (2010) demonstrate that homogeneous cloud assumption (of ice particle size) on the vertical can affect the solar reflected bi-spectral and infrared split-window methods to different extents and, consequently, can lead to significantly different retrievals of effective dimensions and IWC.

The present work deals with thin cirrus clouds which, among ice clouds, are particularly difficult to characterize due to their altitude and low IWC. It aims at (i) improving the knowledge and characterization of vertical properties of cirri, (ii) contributing to the refinement of the interpretation of remote sensing data and (iii) decreasing the uncertainties related to the computations of radiative fields (which, currently, are normally generated assuming homogeneity in the cloud features along the vertical). At this purpose, the vertical backscatter and extinction coefficient profiles within cirrus clouds are investigated. The work is based on active remote sensing data since the investigation of vertical cloud properties using passive remote-sensing measurements is subjected to some limitations. Passive sensors are mainly used to retrieve cloud top height, water phase and bulk optical or microphysical properties (King et al., 2004; Baum et al., 2005a,b). Only recently, a tentative analysis was done by Wang et al. (2009) who developed an algorithm to derive ice particle effective sizes at cloud top and bottom using visible and infrared data of the MODerate resolution Infrared Spectroradiometer (MODIS). The accuracy of the methodology is still low and drastically decreases for clouds with optical depth outside the range 2-15 and particle effective sizes (at the top of the cloud) larger than $80 \mu \mathrm{m}$.

On the other hand, active sensors such as lidars and radars, under certain circumstances, allow a better description of specific vertical properties of cloud layers. Lidar systems are very suitable for observing vertical characteristics of thin ice clouds, while radars mostly miss the detection of cloud layers with small ice crystals, like those found in high-level cirrus clouds that are the subject of the present study.

Current lidars can sense scattering layers with visible OD lower than about 3. Until few years ago, these instruments were mostly ground-based and thus only scattering layers at the zenith of the measuring site could be ob- served. When high clouds are studied using lidar observations, the up-looking measurement configuration shows some disadvantages: both low-level clouds and thick aerosol layers attenuate the lidar pulse, often preventing the possibility of sensing high tropospheric clouds. For this reason data from the Cloud-Aerosol LIdar with Orthogonal Polarization (CALIOP), on-board the Cloud-Aerosol Lidar and Infrared Pathfinder Satellite Observation (CALIPSO) launched into orbit in 2006 by NASA, are used. CALIOP provides continuous global observations of the atmosphere and a new vision of the upper troposphere with focus on thin, high clouds.

CALIOP global observations have been extensively utilized to perform statistical analysis of some cirrus clouds physical parameters. For example, Berthier et al. (2008) used CALIOP lidar, in combination with LITE (Lidar In-space Technology Experiment) and GLAS (Geoscience Laser Altimeter System), to assess the vertical distribution of cloud altitudes on a global extent. Holz et al. (2008) performed a study of the cloud top height comparing the measurements obtained from CALIOP with the ones retrieved from MODIS. Nazaryan et al. (2008) used CALIOP data to investigate the occurrence frequency of cirrus clouds on a nearglobal scale as a function of time, latitude, and altitude. Cho et al. (2008) combined a year of CALIOP and MODIS data to study relationships among cloud phase, cloud height, and cloud optical thickness and to define a methodology for deriving the class of the observed cloud by using only the relation among these quantities. A detailed study of the global distribution of cirrus clouds is done by Sassen et al. (2008) using the Cloud Profiling Radar (CPR) and CALIOP sensors together. They provide a measure of the cirrus clouds occurrence depending on latitude, height, surface type and season for a year of data. Martins et al. (2011) made use of $2.5 \mathrm{yr}$ of CALIOP nighttime data in order to investigate properties of cirrus clouds such as thickness, top altitude and midlayer temperature, and put them in relation to atmospheric dynamics and water vapor. As many others, the above cited works show that one of the advantages offered by CALIOP, with respect to ground-based lidars, is its global and down-looking view of the atmosphere.

The present work, which is focused on CALIOP data in presence of ice clouds in the upper troposphere, provides the first analysis, on a nearly global scale, of the vertical structure of the extinction and backscatter coefficients of cirri and investigates the vertical profile of high, optically thin clouds. Only cirrus clouds with nearly constant top and base altitude in a $5-\mathrm{km}$ field of view are selected for the analysis so that possible artifacts due to geometrical inhomogeneities are removed. Statistics concerning the frequency of occurrence and the cloud physical features of horizontally homogeneous cirrus clouds are provided, for the first time, and compared to those obtained for cirri in general. The vertical extinction profile (ETP) and the vertical backscatter profile (BSP) are strictly related to the vertical distribution of the IWC which, in turn, is fundamental for determining the radiative effect 
and the energy balance of the cloud layer and is a prognostic variable inside Numerical Weather Prediction (NWP) and Climate Models (CM). The main goal of this study is to analyze the statistical features of cirrus clouds vertical extinction and backscatter profiles collected at midlatitudes and tropics over the ocean. A statistic of the shape of the CALIOP BSPs (and ETPs) of cirrus clouds is thus derived. The dependence of the profiles on physical parameters, such as cloud optical depth, cloud geometrical thickness $(\Delta z)$ and temperature, is also investigated and a classification of ice clouds based on the vertical features of the BSPs (and ETPs) is provided.

The paper is organized as follows. In Sect. 2 a description of the data selection and processing is provided. In the same section statistics on frequency of occurrence of horizontally homogeneous cirrus clouds are given and compared to same distributions for iced, optically thin clouds in general. In Sect. 3 annual and seasonal BSPs are computed for night and day and for tropics and midlatitudes. The influence of the main atmospheric and cloud parameters on mean BSPs is studied. Fitting functions, that describe the mean shape of the BSP as function of the cloud geometrical thickness, are derived. Section 4 dwells on the relation of the BSP with ice content profile. The analytical fitting functions, describing the climatological shape of the BSPs, are used as inputs in a forward radiative transfer model to evaluate the impact of the CALIOP-derived IWC profiles with respect to assuming a constant IWC profile. A summary is given in Sect. 5.

\section{Data set and methodology}

Data analyzed in this study cover the full year 2008, so that all the meteorological seasons can be investigated. Two distinct CALIOP products (http://www-calipso.larc.nasa.gov/ resources/calipso_users_guide/data_summaries/) at 5-km horizontal resolution, version 3, are used: (1) the Level 2 cloud layer (CLay) and (2) the Level 2 cloud profile (CPro) files (Anselmo et al., 2007).

The CLay content has column and layer descriptors. The column descriptors contain information on temporal and geophysical location and details on surface type, sunlight conditions and number of cloud layers. Each layer is then described by the layer descriptors, which provide information on spatial and physical characteristics of the scattering layers. The CLay parameters here used are: cloud top and base altitude $\left(z_{t}\right.$ and $\left.z_{b}\right)$, cloud optical depth $(\tau)$, latitude and longitude of the instantaneous field of view (iFOV), UTC time of measurement, layer midpoint temperature and the feature classification flags (see http: //www-calipso.larc.nasa.gov/resources/calipso_users_guide/ data_summaries/layer/\#feature_classification_flags). The midlayer temperatures contained in the CLay data are derived from the GEOS-5 (Goddard Earth Observing System) model and provided by the GMAO (Global
Modeling Assimilation Office) Data Assimilation System (http://gmao.gsfc.nasa.gov/index.php).

The CPro files provide information on the whole atmospheric column and, in particular, contain the measured vertical profiles of particulate backscatter and extinction coefficients. The backscatter coefficient $\left(\beta^{\prime}(z)\right.$, including particulate and molecular components) is derived from the measured attenuated backscatter:

$\beta_{a}(z)=\beta^{\prime}(z) \mathcal{T}^{2}(z)$

where $\mathcal{T}^{2}$ is the two-way transmittance. Note that symbols used in the present work do not correspond with those used in the CALIPSO documentation (references are given throughout the text) and caution should be taken to avoid possible confusion. The derivation of the particulate backscatter coefficient $(\beta(z))$ from the above equation requires estimation of additional quantities (i.e. multiple scattering factor) and ancillary data (i.e. molecular number density and ozone absorption coefficient profiles) whose calculation might lead to errors and uncertainties in the retrieved products, as discussed in http://www-calipso.larc.nasa.gov/resources/pdfs/ PC-SCI-202_Part4_v1.0.pdf. In the present study it is assumed that any measurement uncertainty and error associated with cloud products retrieval do not systematically affect the shape of the BSP (and ETP).

The uncertainties associated with the particulate backscatter and extinction coefficients in the Level 2 data lead to estimate percentage errors of the order of $40-50 \%$ along the cloud body, both for backscatter and extinction coefficient. The percentage errors are usually larger (of a factor 2 or even 3) close to cloud base and top where the backscatter signal might be very low. All the errors are considered random and totally uncorrelated (http://eosweb.larc.nasa.gov/PRODOCS/calipso/pdf/ CALIOP_Version3_Extinction_Error_Analysis.pdf).

Once the particulate backscatter is retrieved, the particulate extinction $(\sigma(z))$ at level $z$ is obtained by multiplying by the appropriate particulate lidar ratio $S_{\mathrm{P}}$ :

$\sigma(z)=S_{\mathrm{P}} \beta(z)$.

Note that in the CALIOP data processing, to derive analytical solutions from the lidar equation, the lidar ratio is constant throughout a feature layer (i.e. a cloud). As a consequence, backscatter and extinction profiles of the considered cirrus clouds have the same shape, thus the same results can be obtained studying either the ETPs or BSPs. The backscatter coefficient profile is derived from the $532 \mathrm{~nm}$ laser data. Its vertical resolution is $60 \mathrm{~m}$ (from ground level up to a height of $20.2 \mathrm{~km}$ ). Horizontal resolution of CPro (and corresponding CLay) data is $5 \mathrm{~km}$, obtained by measures of $15 \mathrm{iFOVs}$ of about $80 \mathrm{~m}$ each.

\subsection{Cirrus clouds selection: reduced database}

The present study is focused on tropical and midlatitude areas. Three latitudinal belts are initially defined: tropics 
Table 1. Conditions used for the selection of clouds in the Reduced Database.

\begin{tabular}{lll}
\hline & Condition & Value \\
\hline (1) & Surface: & sea \\
(2) & Cloud Layer base: & \\
& TRO & $>8 \mathrm{~km}$ \\
& ML & $>5 \mathrm{~km}$ \\
(3) & Cloud Layer top: & $<20 \mathrm{~km}$ \\
(4) & Midpoint Temperature: & $<273 \mathrm{~K}$ \\
(5) & Cloud Type and Phase: & ice clouds \\
& Feature Type & 2 (cloud) \\
& Feature Type QA & 3 (high confidence) \\
& Ice/Water Phase & 1 or 3 (ice) \\
(6) & Cloud Layer: & Upper layer \\
(7) & Cloud Optical Depth: & $0.001<$ OD $<2$ \\
\hline
\end{tabular}

$\left(30^{\circ} \mathrm{S}-30^{\circ} \mathrm{N}\right)$, midlatitudes North $\left(30^{\circ} \mathrm{N}-60^{\circ} \mathrm{N}\right)$ and midlatitudes South $\left(30^{\circ} \mathrm{S}-60^{\circ} \mathrm{S}\right)$. The distinction is done considering the different physical processes that characterize these regions, such as stronger convection in tropical zones, different mean circulation, diverse cloud formation processes (Lynch, 2002; Holton, 2004; Sassen et al., 2008). Data collected in polar regions (above $60^{\circ} \mathrm{N}$ and below $60^{\circ} \mathrm{S}$ ) are excluded from the database since the quasi-polar orbit of the CALIPSO satellite causes oversampling of very high latitude areas.

A reduced database (RD), consisting of data concerning high-level iced clouds only, is derived from the full database containing all the CLay and CPro files of year 2008 (about 20000 files for a total of around 80 million FOVs). CALIPSO ETPs and BSPs are selected and stored into the $\mathrm{RD}$ if all the conditions listed in Table 1 are met. These conditions are applied to ancillary data of the CLay files to obtain a RD consisting of BSPs (and thus ETPs) of high-altitude, optically thin ice clouds over the ocean.

Cloud base threshold altitude is set at $5 \mathrm{~km}$ at both ML and at $8 \mathrm{~km}$ at tropics (TRO) similarly to what done in previous works (Nazaryan et al., 2008; Eguchi et al., 2007). Clouds with optical depth larger than $10^{-3}$ and less than 2 only are selected. As in Martins et al. (2011) cloud layers with optical depth smaller than $10^{-3}$ are removed because they are considered to be below the threshold confidence of lidar data and can be attributed to noise. Maximum optical depth allowed is set at 2.0 so that the full cloud body is sensed by the lidar ray and total attenuation is not reached.

The selection of ice phase clouds only is obtained through the use of multiple requirements: altitude of base and top (conditions 2 and 3 of Table 1), midpoint temperature (i.e. the temperature at the geometrical middle-point of the cloud thickness, condition 4) and above all the use of CALIOP Feature Classification Flags (condition 5). As in Nazaryan et al. (2008) only those cloud layers from the CALIPSO data
Table 2. Cirrus clouds cover obtained from the reduced database, RD.

\begin{tabular}{lccc}
\hline & Day & Night & Total \\
\hline Midlatitudes North & $36 \%$ & $35 \%$ & $36 \%$ \\
Tropics & $35 \%$ & $39 \%$ & $37 \%$ \\
Midlatitudes South & $32 \%$ & $30 \%$ & $31 \%$ \\
\hline
\end{tabular}

record that are identified by the CALIOP cloud-aerosol discrimination (CAD) algorithm as clouds with high level of confidence (Feature Type QA equals 3) are selected. To avoid the presence of supercooled water clouds in our reduced database further conditions provided by CAD algorithm and concerning the cloud phase are used: the Ice/Water Phase field of the Feature Classification Flags must be either randomly or horizontally oriented ice particles.

Requirement number 6 in Table 1 ("Cloud Layer" equals "upper-layer" cloud) is introduced to account for multiplelayer clouds. When more than one scattering layer in the same FOV is detected, only the first one (i.e. the highest) is considered, and its backscatter (extinction) profile is recorded into the RD. This criterion allows the inclusion of the BSP of the upper layer of condensate into the RD when conditions of multiple-layer clouds are found.

When all the conditions listed in Table 1 are met, the BSPs contained in the CPro file are copied in the RD. Each selected profile is recorded together with the corresponding ancillary data taken from the CLay. Repetition of the same procedure for all the FOVs contained in the CPro files of year 2008 completes the RD. The clouds contained in the RD are simply referenced as cirrus clouds or cirri in what follows.

Some basic statistics concerning the frequency of occurrence of cirrus clouds selected for the RD are given in Table 2. The table shows that, on average, cirrus clouds cover more that $30 \%$ of ocean FOVs from $60^{\circ} \mathrm{S}$ to $60^{\circ} \mathrm{N}$ with a maximum in the tropical area. Similarly to what noted by Sassen et al. (2008), Martins et al. (2011) and Nazaryan et al. (2008) the occurrences are higher in the northern hemisphere than in the southern one. This feature is also found within the tropics where differences between the northern belt and the southern one are of the order of $10 \%$. Due to some differences in the selection criteria, the absolute values of the cirrus clouds frequency do not exactly match those found by previous authors; nevertheless the general features of the statistical ensemble are preserved, i.e., cirrus clouds during nighttime have higher occurrences in tropical areas and lower occurrences in the midlatitude belts with respect to what was found during daytime in the same areas, as in Nazaryan et al. (2008).

The main physical and optical characteristics of the cirrus clouds selected for the RD are described by means of percentage distributions in Fig. 1 for tropics and midlatitudes and for nighttime and daytime separately. It is shown that at 

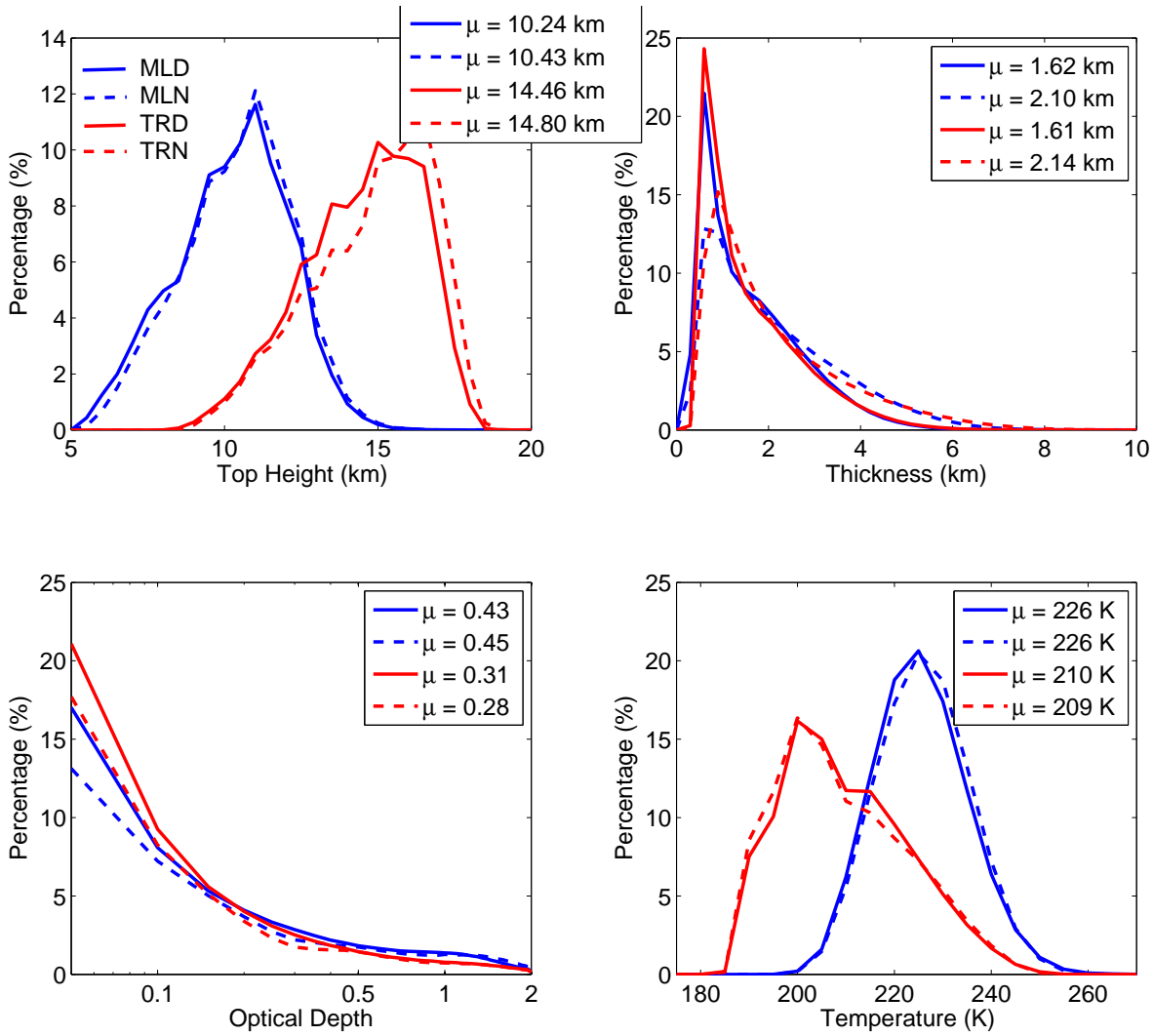

Fig. 1. Top height (upper left panel), geometrical thickness (upper right), optical depth (lower left) and midlayer temperature (lower right) distributions for the cirrus clouds selected in the Reduced Database (see Table 1). Midlatitude data are in blue and tropics in red. Dashed lines are for nighttime cirrus clouds (MLN and TRN) while solid lines are used for daytime (MLD and TRD). For each distribution the mean value $\mu$ is also reported. Lower left panel is in Logarithmic-Linear scale

tropics the mean top altitude is $14.5 \mathrm{~km}$ during the day and $14.8 \mathrm{~km}$ during the night while at ML it is about $10.2 \mathrm{~km}$ and $10.4 \mathrm{~km}$ respectively for day and night. These values are in good agreement with those found by Nazaryan et al. (2008) and Martins et al. (2011). The geometrical thickness distributions are highly peaked at $0.8 \mathrm{~km}$ at day and at around $1 \mathrm{~km}$ at night for both latitudes denoting the widespread occurrence of thin cirri. The mean thickness is larger during the night than at daytime with very similar values for both latitudes (about $2.1 \mathrm{~km}$ at night versus $1.6 \mathrm{~km}$ during the day). Distributions with higher and thicker cirrus clouds are then found during the night than at daytime. It is suggested that the nighttime-daytime differences might be related with the higher levels of daytime noise. In fact, if the backscatter signals near cloud top and base were below the noise level, the cloud boundaries detected by the algorithm will be closer to the center of the cloud than is actually the case. As a consequence, the detected cloud top heights will be lower and thicknesses smaller than is actually the case. The thickness distribution at tropics is in accordance with Martins et al. (2011) while at ML some differences are found, possibly due to the very stringent condition applied on cloud midlayer temperature (maximum at $233 \mathrm{~K}$ ) by the cited authors. The OD distributions show the high predominance of optically thin cirrus clouds (especially during nighttime) and a monotonic and rapid percentage decrease from $10^{-3}$ to 2 optical depth. The lower-right panel of Fig. 1 shows the distributions of the midpoint cloud temperature. For the midlatitude case both day and night midlayer temperature distributions are almost equally spread around the mean value $(226 \mathrm{~K})$ while for the tropical case the distributions for night and day data show (again very similar and with a mean value of around $209 \mathrm{~K}$ ) a higher degree of asymmetry and a longer tail toward the larger temperature with respect to the mode value.

\subsection{Definition of the final database}

Backscatter (and extinction) profiles contained in the RD refer to cirrus clouds covering a wide range of vertical extents (from few hundreds $\mathrm{m}$ up to $8 \mathrm{~km}$ ) and optical depths (from $10^{-3}$ to 2 ).

The cloud geometrical thickness is proportional to the integer number of the backscatter (or extinction) coefficient values used to describe the layer in the CPro files that have a vertical resolution of $60 \mathrm{~m}$. On the other hand, the total 
layer optical depth influences the magnitude of the layerbackscattered signal. Since we are interested in deriving statistical features of the shape of the BSPs (and ETPs) and investigating if relations with some of the main atmospheric parameters (included cloud's OD and $\Delta z$ ) exist, data are subjected to a process of rescaling and normalization. Thus a comparison among the shape of profiles, which originally would differ for the total amount of energy backscattered to the sensor and for the number of scattering levels, is made possible.

Moreover, a procedure that discards all the FOVs with non-uniform cirrus clouds geometrical features is also applied. The operation is performed to avoid the possibility that horizontal variations in cloud top and base altitude, within the 5-km resolution CALIOP data, might affect the averaging process of the shape of the BSPs.

The CALIOP parameter QC concerning the quality of the extinction retrieval (http://www-calipso.larc.nasa.gov/ resources/calipso_users_guide/data_summaries/profile_data. php\#extinction_qc_flag_532) is not utilized in this study. It is in fact demonstrated (detailed tests have been performed but results are not shown here) that the differences in mean BSPs obtained by averaging data of the FD with QC equal to 0 or 1 (highest quality retrieval) or alternatively with $\mathrm{QC}$ free to assume any possible value are of minor importance.

These operations, described in detail in the two following subsections, lead to the creation of a new database called the Final Database (FD) over which the analysis of the BSPs (and ETPs) is performed.

\subsubsection{Homogenization}

With the term homogenization the operation of selecting cirrus clouds with horizontally homogeneous top and base altitudes is meant.

The 5-km horizontal resolution Level 2 data products (CLay and CPro) used in the present study are obtained by applying sophisticated algorithms

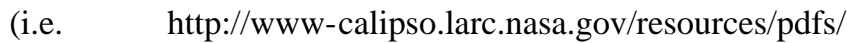
PC-SCI-202_Part4_v1.0.pdf, http://www-calipso.larc.nasa. gov/resources/pdfs/PC-SCI-202_Part2_rev1x01.pdf) to averaged Level 1, full resolution, attenuated backscatter profiles, in order to obtain high signal-to-noise ratios (SNR). Since horizontal inhomogeneities could introduce artifacts in the analysis of the shape of the BSPs, the non-uniform 5-km horizontal resolution FOVs are discarded from the database.

The criterion used to define the geometrical horizontal homogeneity within the 5-km horizontal resolution CALIOP FOVs is based on the analysis of cloud top and base height retrieval at higher resolution (i.e. $1 \mathrm{~km}$ ). Cloud top and base altitudes stored in the Level 2 CLay at 1-km horizontal resolution are collocated with the same products of the $5-\mathrm{km}$ CLay files selected in the RD. For each 5-km FOV, the cloud top and base heights $\left(z_{t}\right.$ and $\left.z_{b}\right)$ are respectively compared to the mean cloud top and base heights $\left(\overline{z_{t}} 1 \mathrm{~km}\right.$ and $\left.\overline{z_{b}} 1 \mathrm{~km}\right)$ obtained by averaging the five cloud top and base products of corresponding 1-km CLay files. The results are finally divided by the cloud thickness, as shown in the following equations:

$$
\begin{aligned}
& \mathcal{Z} t=\frac{\left|z_{t}-\overline{z_{t}} 1 \mathrm{~km}\right|}{\Delta z} \\
& \mathcal{Z} b=\frac{\left|z_{b}-\overline{z_{b}} 1 \mathrm{~km}\right|}{\Delta z}
\end{aligned}
$$

It is assumed that a horizontally uniform cirrus cloud fills the observed 5-km FOV whenever both the conditions $\mathcal{Z} t<$ 0.1 and $\mathcal{Z} b<0.25$ are met. The condition on the cloud top homogeneity is more stringent than the one on cloud base since its derivation is performed on data with higher SNRs.

The number of profiles contained in the RD and discarded after applying the requirement on the horizontal homogeneity is significant and corresponds to about $75 \%$ at $\mathrm{ML}$ and $83 \%$ at tropics, revealing that, on a $5-\mathrm{km}$ horizontal resolution scale, less than one-fourth of cirrus clouds show homogeneous geometrical features.

\subsubsection{Rescaling and normalization of Extinction and Backscatter Profiles}

The rescaling process is obtained by using a linear interpolation. The original BSPs (and ETPs) are rescaled to a standard length of 60 points ( $I$ height indexes). This means that, regardless of the geometrical thickness of the cloud, the rescaled BSPs (ETPs) are described by 60 values of the backscatter (extinction) coefficient. The height indexes are equally spaced.

The backscatter coefficient at height index $h, \beta(h)$, assumes different values which are strongly correlated with the cloud-layer optical depth. A normalized backscatter coefficient, $\beta_{n}(h)$, is obtained by dividing the level-backscatter profile by the total area of backscatter profile (computed from cloud-base to cloud-top altitude). Since this operation is performed after the height rescaling at $I=60$ equally spaced points, the total area is obtained by a simple summation. Using the trapezoidal rule the normalized backscatter coefficient at level (height index) $h$ is written as:

$\beta_{n}(h)=\frac{\beta(h)}{\frac{\beta(1)+\beta(I)}{2}+\sum_{h=2}^{I-1} \beta(h)}$

At this stage, normalized backscatter profiles from cirrus clouds with different ODs are comparable and stored in the final data set. In Fig. 2 an example of the normalization and rescaling process, for two randomly selected BSPs, is shown.

Along with the normalization procedure an additional data filtering process is applied to the database. The process is based on the analysis of $\beta_{n}(h)$. This quantity should be positive for all the 60 height indexes $h$, unless for data having a SNR smaller than 1 or for bad instrument calibration. 

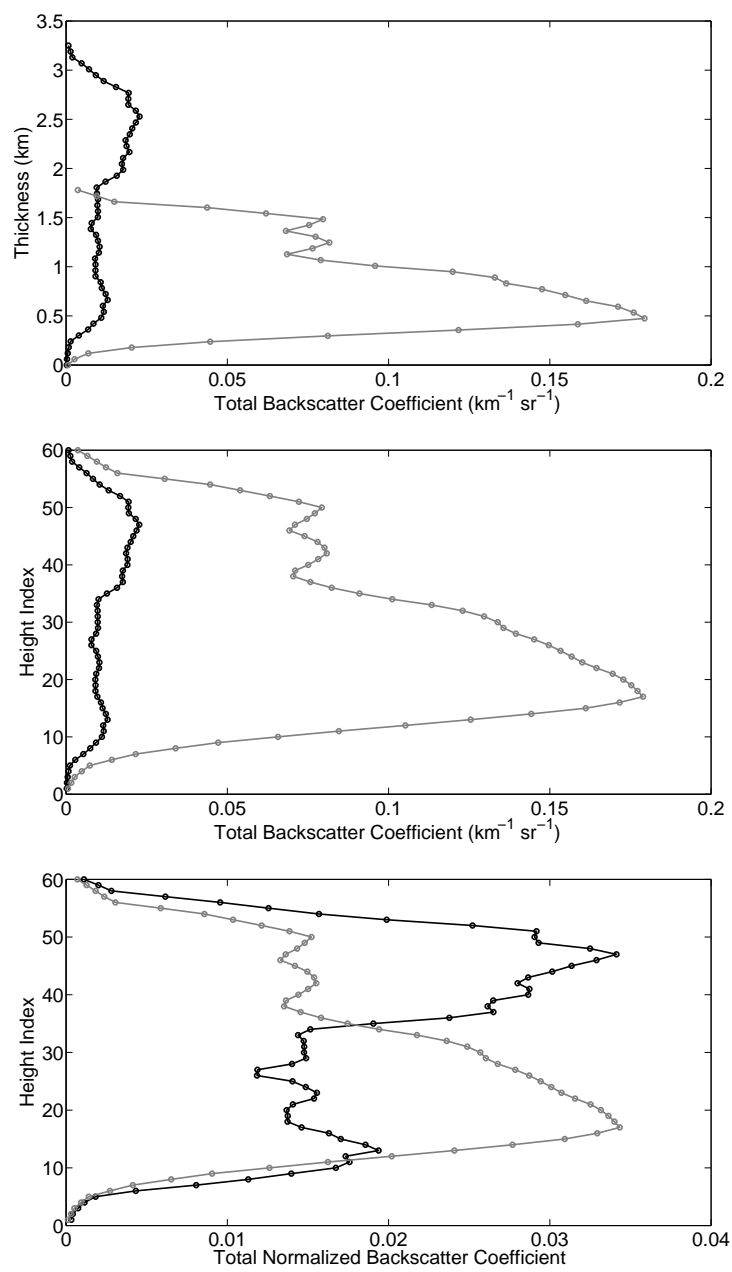

Fig. 2. Pre-processing operations performed on two randomly chosen BSPs. Upper panel: CALIOP BSPs are compared without any manipulation. Middle panel: the backscatter profiles are rescaled to a standard number of points (60 height indexes). Lower panel: the two BS profiles are normalized to unit area as functions of the height index.

In this regard, BSP data derived from CPro files and selected in the RD may show two kinds of problems: (1) profiles with some negative backscatter values and (2) profiles with some zero backscatter values between the cloud-top and cloud-base height. If one of the two cases occurs the profile is discarded from the final data set.

\subsubsection{Statistical features of the Final Database}

The analysis of the shape of BSP is performed on the ensemble of $\beta_{n}(h)$ contained in the FD. The subscript $n$ is omitted in the following, and for sake of simplicity, this quantity is called BSP. Since the shape of BSPs and ETPs is the same, only results concerning BSPs are shown. Cloud geometrical depths, BSPs (and ETPs) total areas (see Eq. 5) and all the
Table 3. Cirrus clouds cover obtained from the final database, FD.

\begin{tabular}{lccc}
\hline & Day & Night & Total \\
\hline Midlatitudes North & $8 \%$ & $10 \%$ & $9 \%$ \\
Tropics & $6 \%$ & $7 \%$ & $6 \%$ \\
Midlatitudes South & $8 \%$ & $9 \%$ & $8 \%$ \\
\hline
\end{tabular}

other ancillary data are recorded in the final database, so that the original BSPs (and ETPs) might be reconstructed at any time.

The FD consists of about 1.5 million cirrus cloud cases: about $43 \%$ of the total is found in the tropics and the remaining at ML (37\% in the ML South and $20 \%$ in the ML North). In Table 3 the percentage of CALIOP FOVs selected for the FD, with respect to the total number of FOVs observed over the sea at the same latitudinal areas in 2008, is reported. Table 3 gives an idea of the occurrence frequency of horizontally homogeneous cirrus clouds over the ocean which are detected in approximately $8 \%$ of the $5-\mathrm{km}$ horizontal resolution CALIOP FOVs. Statistics for daylight and nighttime occurrence are also considered. With respect to those reported in Table 2 and referring to the RD (that concerns cirrus clouds in general), the occurrence frequencies reported in Table 3 are drastically reduced. In particular, high percentages of optically (and geometrically) thin cirrus clouds present in the RD are excluded from the FD.

This is also shown in Fig. 3, which highlights the general physical and optical features of the geometrically homogeneous cirrus clouds contained in the FD for tropics and ML and for day and nighttime separately. The frequency distributions of cloud top height (upper left panel) are very similar to those shown in Fig. 1. Only slight shifts toward higher altitudes at ML and toward lower altitudes at tropics for the FD are observed. The largest changes, with respect to what shown for the RD, are found in the geometrical thickness and optical depth distributions. The occurrence frequencies of geometrically and optically thin cirrus clouds in the FD are strongly reduced with respect to what found for the RD. The mean cloud $\Delta z$ is 2.2 and $3.0 \mathrm{~km}$ for ML day and night respectively and 2.3 and $3.3 \mathrm{~km}$ for tropical day and night, which means at around a $50 \%$ increase with respect to what was found considering all cirrus clouds. The ODs frequency distributions are also drastically changed and mean ODs are shifted to higher values (about 0.96 for ML and 0.83 for tropics). Some changes are also found in the midlayer temperature, which is increased (about $1 \mathrm{~K}$ for the ML and $6 \mathrm{~K}$ for the tropics) with respect to what found for the RD.

In Table 4 some statistics concerning the occurrence of overlapped clouds are given for day and night. The table shows that, at midlatitudes, more than $50 \%$ of the FOVs that are selected in the FD consists of non-overlapped clouds. The number increases at tropics and involves about twothirds of clouds of the FD when day and night cases are 

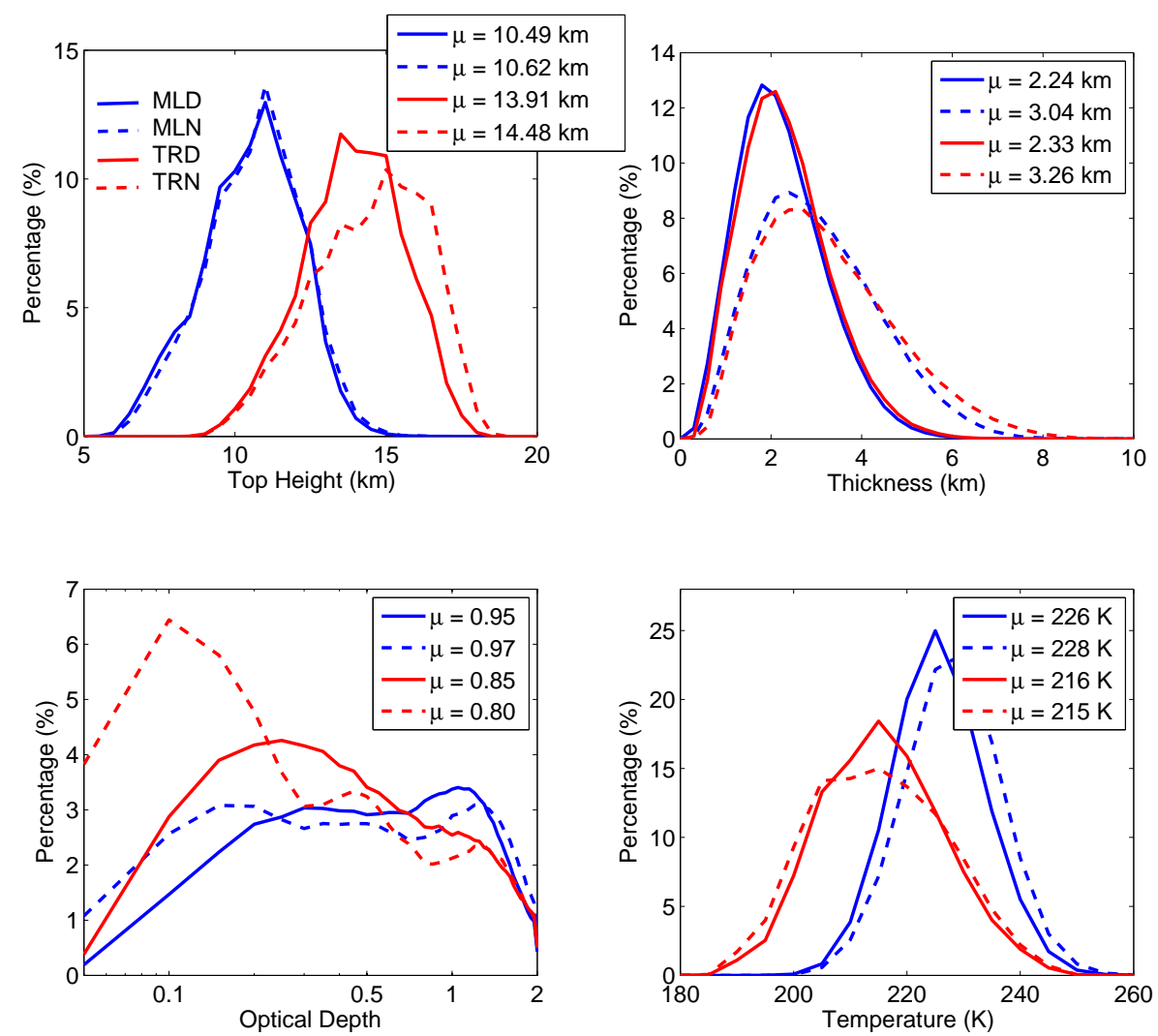

Fig. 3. Same as in Fig. 1 but for geometrically homogeneous cirrus clouds (the Final Database).

Table 4. Coud overlap in the FD.

\begin{tabular}{lccc}
\hline \# of layers in the FOV: & 1 & 2 & $>2$ \\
\hline ML N day & $66 \%$ & $20 \%$ & $14 \%$ \\
ML N night & $55 \%$ & $26 \%$ & $19 \%$ \\
TRO day & $70 \%$ & $20 \%$ & $10 \%$ \\
TRO night & $60 \%$ & $24 \%$ & $16 \%$ \\
ML S day & $62 \%$ & $22 \%$ & $16 \%$ \\
ML S night & $51 \%$ & $24 \%$ & $25 \%$ \\
\hline
\end{tabular}

considered together. Note that for the RD the percentage of overlapped cirrus clouds is significantly higher (not shown here).

\section{Analysis of CALIOP vertical Backscatter and Extinc- tion Profiles}

A detailed statistical analysis of the shape of the BSPs is performed accounting for the whole FD (horizontally homogeneous cirrus clouds). The influence of cloud physical parameters (mainly geographical location, cloud optical depth, geometrical thickness and temperature) on cloud vertical features of the BSP is investigated. This requires the definition of a mean vertical BSP and, for each height index, a mean value of the backscatter coefficient is derived. Since the processes of averaging are performed on the full FD, which includes clouds with very different physical and geometrical properties, the problem of the shape of the distribution of data around the mean (for each altitude level) arises. For the present study, at each altitude level (height index), the definition of a mean backscatter (BS) value makes sense only if the data are approximately symmetrically distributed around a maximum value.

All the means obtained in this work are such that the distribution of the backscatter values at each height index is unimodal and approximately symmetric. As a consequence, a mean value of BS (at each level) can be defined unambiguously and used to derive the full average profile.

\subsection{Definition of the Difference Index}

To evaluate the results of the analysis, an index quantifying the distinction between two (mean) BSPs is also needed. A Difference Index, $\mathcal{I}_{\Delta}$, is then defined as:

$\mathcal{I}_{\Delta}=\frac{\sum_{h=1}^{I}\left|\beta(h)-\beta^{*}(h)\right|}{\frac{\beta(1)+\beta(I)}{2}+\sum_{h=2}^{I-1} \beta(h)} \cdot 100$ 


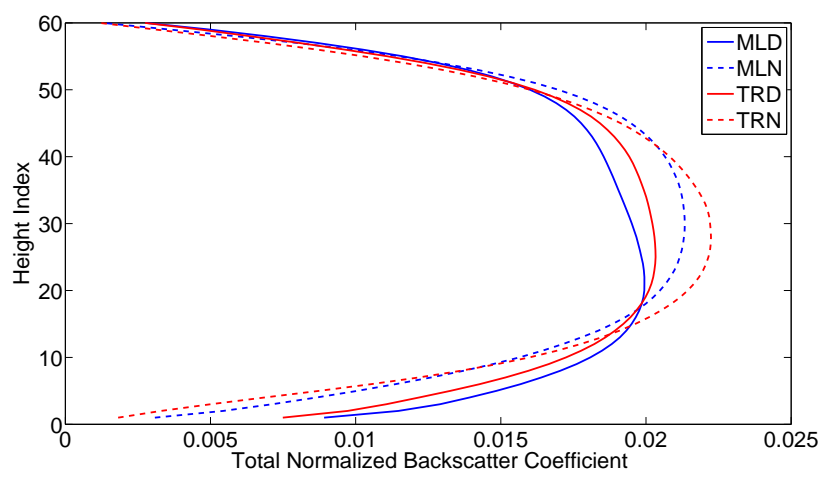

Fig. 4. Annual mean backscatter profile for midlatitude (blue) and tropical (red) cirrus clouds at daytime (solid lines) and nighttime (dashed lines).

where $\beta(h)$ is a reference $\mathrm{BSP}$ and $\beta^{*}(h)$ is the BSP that has to be evaluated against the reference one. $I$ is again the total number of points along the cloud height (60). The difference index is preferred to other indexes (such as the squared differences) because of its simple interpretation. In fact, it represents the percentage value derived from the ratio of the area between the two BS profiles and the total area of the mean reference BSP. Equation (6) can be simplified noting that the denominator (that is the area of the reference BSP) is equal to unity, as a consequence of the normalization of all the profiles contained in the final database.

\subsection{Annual and seasonal averages of the backscatter profiles}

Due to appreciable differences found in the mean physical characteristics of cirrus clouds measured during night and daylight hours (Fig. 3), BSPs collected at nighttime and daytime are analyzed separately. Measurements obtained at midlatitudes and tropics are studied individually too.

Annual means of the shape of the BSPs are given as functions of the 60 height indexes: height index number 1 is cloud base and number 60 is cloud top. Results are plotted in Fig. 4 and show smooth curves with a single maximum BS value located close to cloud midlayer. Very similar profiles for tropics and ML, both at daylight and at nighttime, are observed, thus showing a minor influence of latitude on annual mean BSPs. Note that the distributions of geometrical thickness for homogeneous cirrus clouds at ML and at tropics are very similar while some differences are observed in the cloud top height, optical depth and temperature distributions (see back at Fig. 3).

In contrast, the differences between mean BSPs measured during the night (dashed curves in Fig. 4) and during the day (solid lines) are significant. A broader mean BSP shape in case of daylight cirri is observed with respect to nighttime. In terms of $\mathcal{I}_{\Delta}$ the differences are of the order of $10 \%$ for both latitudes. Physical characteristics reported in Fig. 3 showed that the main differences between night and day distributions are found in the cloud geometrical thickness, suggesting a possible influence of this parameter on the shape of the CALIOP BSP.

From Fig. 4 it is also observed that the mean value of the normalized BS coefficient measured at cloud base during night is lower than that measured during daylight hours. This result can be interpreted either as a CALIOP higher ability to detect cloud base during the night than day due to higher SNRs or as a physical signal that would mean that, on average, sharper cloud base edges are found during the day.

The averaging process is also applied to monthly data, around 25000 cirrus clouds profiles per month. Results (not plotted here) show very little variability on the shape of the BSP with the period of the year. The maximum $\mathcal{I}_{\Delta}$ value found when monthly mean BSPs are compared to the corresponding annual means is less than 5.5\% at ML (North and South ML are studied separately in this regard to highlight the seasonal signal) and less than $2.7 \%$ at tropics (for both North and South tropical belts).

Despite the small difference index, the two main climatic regions (ML and tropics) are studied separately in the next subsections that are focused on the investigation of possible relations among the shape of the BSP and cloud physical and thermodynamic parameters. Due to the minimal seasonal variability observed in monthly mean BSPs, all data presented in what follows are averaged on a full year period.

\subsection{Geometrical thickness and Backscatter Profile}

In order to investigate the influence of the cloud geometrical thickness on the shape of the BSP, annual averages of the CALIOP BS are computed for six subsets of the FD regarding cirrus clouds with different vertical extensions (one interval every step of $1 \mathrm{~km}$ up to a value of $5 \mathrm{~km}$ and one interval for $\Delta z$ larger than $5 \mathrm{~km}$ ). The amplitude of the intervals is chosen so that at least 2000 profiles are selected for each subset. In Appendix A a methodology that derives the minimum number of profiles per subset, which is required to have a statistically relevant ensemble (for subsets of the whole FD), is described.

Results for midlatitudes and tropics are shown in Figs. 5 and 6 for nighttime and daytime respectively. It is observed that, for all the cases presented in the figures, the maximum of the BS profile is close to cloud top for thin cirrus clouds and moves towards cloud base as the cloud geometrical depth increases. For each panel of the figures, the difference indexes, which evaluate the diversity of each curve with respect to the mean BSPs (for midlatitudes and tropics and for night and day, as already shown in Fig. 4), are computed. Results show a great variability among the mean BSPs: $\mathcal{I}_{\Delta}$ is at maximum of about $38 \%$ for midlatitudes and tropics during the night and $36 \%$ during the day. The variability among mean BSPs of cirrus clouds of selected geometrical thickness 

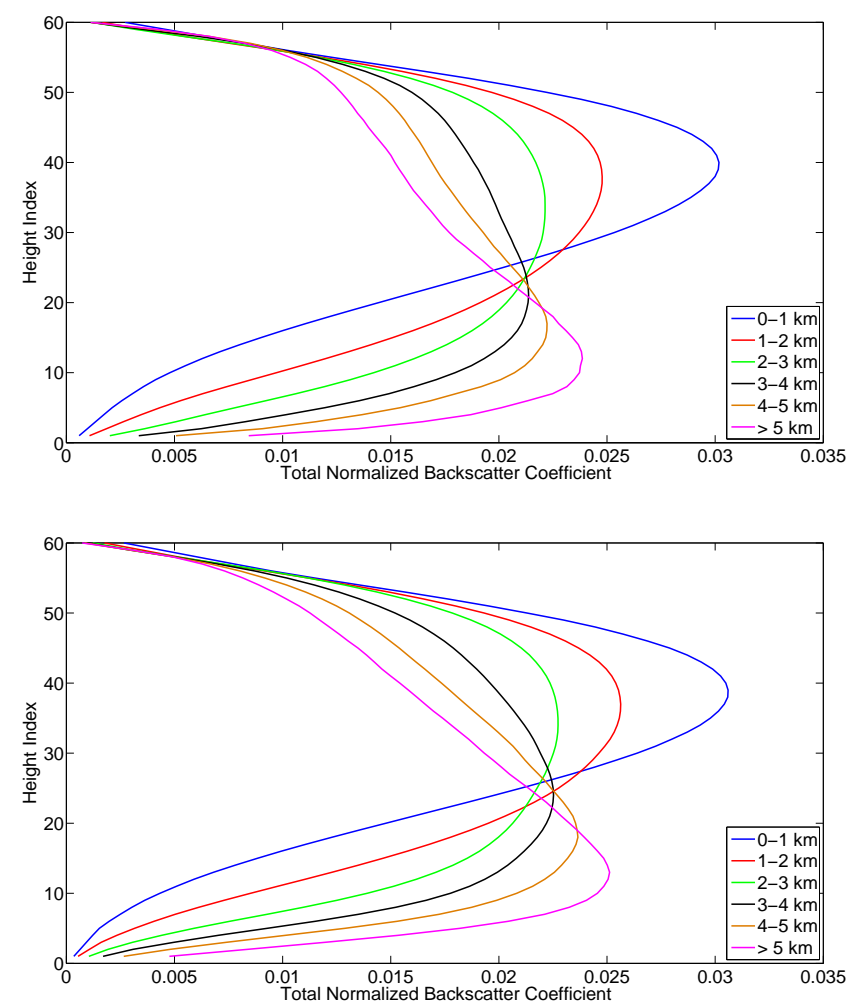

Fig. 5. Cirrus clouds mean backscatter profiles for multiple thickness intervals. Data refer to nighttime. In the upper panel the backscatter profiles of midlatitude clouds are plotted as functions of height indexes. The lower panel shows profiles for the tropical case.

intervals suggests a strong relation between cloud depth and the shape of the BSP.

The mean backscatter profiles plotted in Figs. 5 and 6 are analyzed as distributions along the height-index axis and their skewnesses are calculated. Results show that negative skewness values are found in case of night clouds with $\Delta z$ less than $3 \mathrm{~km}$ both at tropics and ML, while positive ones are observed if the $\Delta z$ is larger than $3 \mathrm{~km}$. For the daylight case the geometrical vertical extent defining the ensembles with negative or positive skewness is $2 \mathrm{~km}$ both for midlatitude and tropical cirrus clouds. Note that a negative skewness value means a BS maximum placed in the upper part of the cloud while a positive one indicates a BS maximum in the lower part of the cloud.

Differences between BSPs of midlatitude and tropical data for cirrus clouds belonging to the same interval of geometrical thickness are minor but appreciable (difference indexes are about $6.5 \%$ for daylight and $8.5 \%$ for nighttime data respectively). Differences of the same order of magnitude are found also when tighter intervals of geometrical thickness are considered (i.e. intervals of width of $0.5 \mathrm{~km}$ or less). Very narrow $\Delta z$ intervals are used to avoid the possibility that different distributions of cloud depth within each interval might
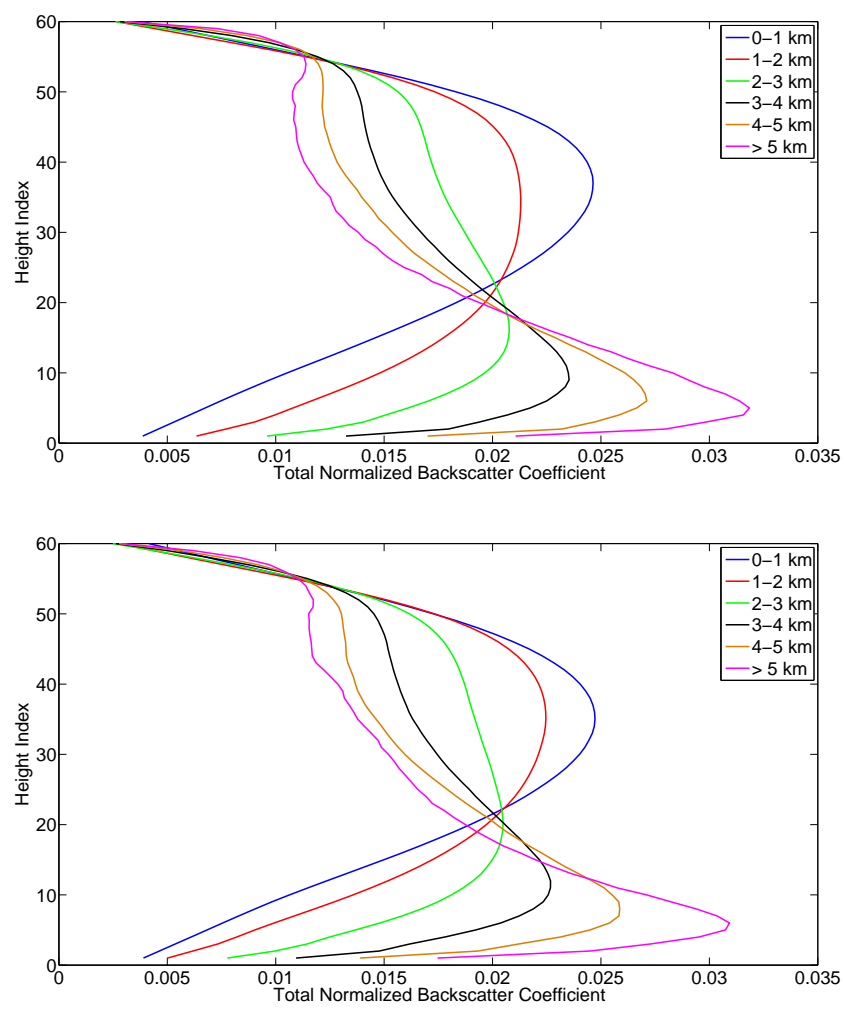

Fig. 6. Same as Fig. 5 but for daylight hours.

influence the results of the comparison among data collected at the two latitudes. The discrepancies found between midlatitude and tropical mean BSPs cannot, thus, be explained in terms of cloud geometrical thickness only and it implies that other parameters, characterizing differently midlatitude and tropical cirrus clouds, might influence the shape of the CALIOP BSP.

In our analysis only cloud bulk parameters, such as optical depth and temperature, are investigated while vertical variation of microphysical features (i.e., PSD or particles habit type) are not considered since no information is available for the cases analyzed in the present study. In this regard, note that, due to the environmental difficulties of collecting these kind of data, only rare and sparse measurements are accessible. Comparison between in situ data collected for tropical and midlatitude cirri show that PSDs measured in ice clouds associated with deep convection exhibit an exponential size distribution behavior, while, typically, midlatitude cirri display narrower PSDs (Heymsfield and Miloshevich, 2003; Baum et al., 2005a,b; Sassen et al., 2008). Nevertheless, it is the vertical variation of the PSD or of the particles' habit that might influence the shape of the BSP; unfortunately, in this regard, available data are few, for certain aspects in contrast among them and not easily interpretable in this context (Heymsfield and Miloshevich, 1995; Lawson et al., 2006). It is worth noting again that, in the CALIOP 
data processing, the lidar ratio is constant throughout a feature layer, meaning that variations of the PSD or the habit type are not accounted for.

Nighttime and daytime data show large discrepancies even when cirrus clouds within the same thickness interval are compared (dashed curves plotted in the panels of Fig. 5 with the corresponding solid lines plotted in Fig. 6). In this case the $\mathcal{I}_{\Delta}$ is of the order of $20 \%$ for both latitudes. This again suggests that possibly different physical features characterizing the two cases might influence the shape of the BSP. The influence of optical depth and temperature on cirrus cloud BSPs is the topic of the next two subsections.

\subsection{Optical depth and backscatter profile}

Similarly to what was done for investigating the relation between cloud BSP shape and geometrical thickness, an analysis concerning the cirrus optical depth and the backscatter profiles shape is performed. The final database is divided into multiple subsets containing cirrus clouds with different $\tau$. For each subset a mean BSP is computed at tropics and midlatitudes and for data collected during the night and day separately. Results (not shown here) are very similar to what obtained previously in the analysis concerning the geometrical thickness and BSPs relation. In fact, the maximum in the shape of the mean BS curve is close to the cloud top for clouds with very small $\tau$ and to the base for the optically thickest clouds of the ensemble (FD). In terms of skewness the BSP mean curves show negative values for OD values less than approximately 1.3 at night and 0.8 at day. Nevertheless, in the present analysis, the dependence between the cloud BSP shape and its optical depth is of minor intensity with respect to the one found for the geometrical thickness. In fact, $\mathcal{I}_{\Delta}$ computed for the mean BSP of each OD interval and the corresponding annual average is at maximum of $22 \%$ (it was almost $40 \%$ in the analysis concerning $\Delta z$ ). Furthermore, some exceptions are found to the rule describing the general behavior (BSP maximum moves toward cloud base as $\tau$ increases).

A correlation exists between cloud geometrical thickness and optical depth. An example of this is shown in Fig. 7 where cloud optical depth is plotted as function of cloud geometrical thickness for data collected in the tropical and midlatitude regions and during the day or at night (the four red and blue lines in the plot). The curves show a monotonic increase of the averaged OD value for any increase in the mean cloud $\Delta z$ considered. The green line in the same figure is obtained for the whole FD without distinction between day and night or latitudinal belt. A parameterized formula that describes the green curve of Fig. 7 is given in Eq. (7):

$\tau=a_{1} \cdot \Delta z^{b}+a_{2}$

where $a_{1}=-2.83, b=-0.1955$ and $a_{2}=3.28$.

The above $\tau-\Delta z$ relation simply parameterizes the mean OD value for a given cloud geometrical thickness when ho-

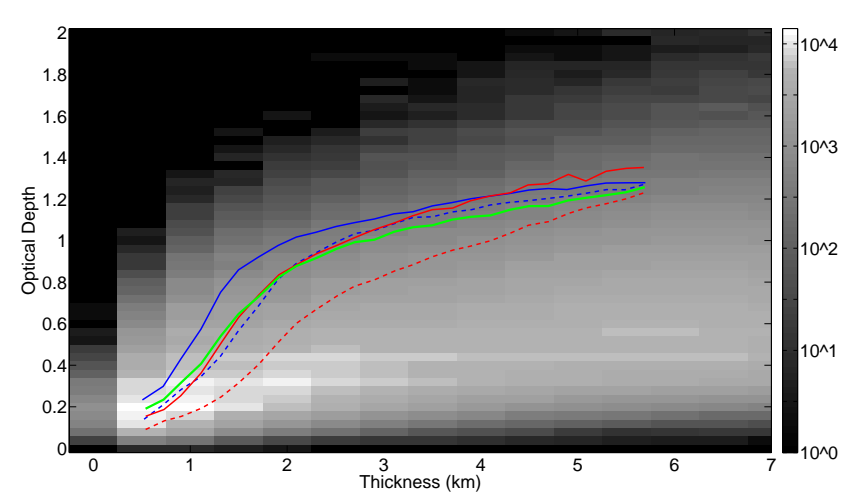

Fig. 7. Optical depth of geometrically homogeneous cirrus clouds is plotted as a function of mean cloud geometrical thickness for ML (blue) and tropics (red) and for day (solid line) and night (dashed line). The green line is the weighted mean of the red and blue curves and represents the mean of the whole Final Database. The curves are plotted above the frequency of occurrence (in grayscale) for the FD as function of $\Delta z$ and $\tau$.

mogeneous and optically thin $(\tau<2)$ cirrus clouds are considered. Equation (7) holds for $\Delta z$ varying from 0.5 to $5.8 \mathrm{~km}\left(R^{2}=0.984\right)$. The spread of the OD distribution around the mean for each geometrical thickness is provided by Eq. (8) $\left(R^{2}=0.973\right)$ :

$\sigma_{\tau}=c_{1} \cdot e^{d_{1} \Delta z}+c_{2} \cdot e^{d_{2} \Delta z}$

where $c_{1}=0.6138, c_{2}=-0.0792, d_{1}=-0.9944$ and $d_{2}=$ -1.5090 .

In Fig. 7 the curves are superimposed on the frequency of occurrence of homogeneous cirrus clouds as function of the $\tau$ and $\Delta z$. The frequency of occurrence highlights the large number of cirrus clouds with geometrical depth between 1 and $4 \mathrm{~km}$ and gives an idea of the distribution of cirrus clouds $\mathrm{OD}$ as function of geometrical thickness and vice versa.

As a conclusion, since a relationship between mean BSPs and geometrical thickness exists it is plausible that also a relationship (even if weaker) exists between BSP shape and $\mathrm{OD}$ as a consequence of the $\tau-\Delta z$ dependence.

In order to verify that the shape of the BSP is mainly driven by cloud geometrical thickness and that the BSP-OD relation is only a second order effect, an analysis concerning the influence of OD value on the shape of the BSP must be applied to data from which the geometrical thickness dependence is removed (or at least limited). In this regard, the relation BSPOD is investigated for cirrus clouds with nearly the same $\Delta z$ but varying $\tau$. In the upper panel of Fig. 8 the BSPs of midlatitude cirri during nighttime are computed for six optical depth intervals and for fixed geometrical depths (only clouds with vertical extension from 2.1 to $2.5 \mathrm{~km}$ are selected so that a large number of cases is accounted for). Results show a limited variability in the shape of the BSP with changing OD value. The largest value of $\mathcal{I}_{\Delta}$ found for the curves plotted in the upper panel of Fig. 8 is $15 \%$. It is observed that the 

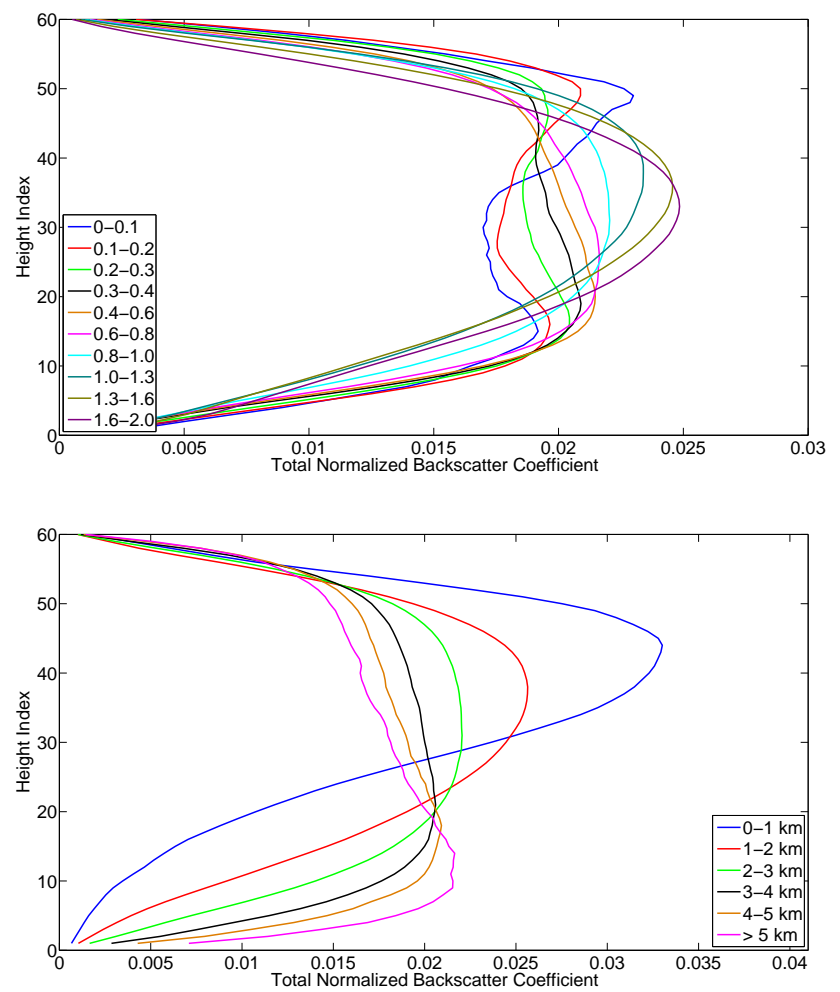

Fig. 8. Upper panel: annual mean BSPs of cirrus clouds for varying optical depth and geometrical thickness between 2.1 and $2.5 \mathrm{~km}$. Lower panel: annual mean BSPs for cirrus clouds with nearly the same optical depth (0.8-1.0) and multiple geometrical thicknesses. For both panels ML at nighttime are considered.

maximum of the BSP curves slightly moves from top of the cloud toward the cloud base as $\tau$ increases. Also, the higher is the OD and the narrower is the distribution of the backscatter values around the maximum (and lower values near cloud top and base). This behavior is observed for both day and night profiles and for both the climatic regions.

As a comparison with the results shown in the upper panel of Fig. 8 the lower panel shows BSPs of midlatitude cirrus clouds at nighttime computed for varying geometrical thickness (six intervals) but nearly constant optical depth (0.8-1.0, so that the number of cases considered is maximized). It is demonstrated that a strong dependence between the shape of the BSP and the cloud thickness exists and that the maximum of the BSP curves shifts from cloud top to cloud base with increasing cloud geometrical depth. In this case the maximum $\mathcal{I}_{\Delta}$ among the curves is of $48 \%$. It is found that, for both latitudes and day and night, the difference indexes found when $\Delta z$ is varied and $\tau$ is fixed are larger (by a factor between 1.5 and 3.2) than those found for varying optical depth intervals and fixed geometrical thickness.

Results show that the cirrus cloud optical depth has a smaller but appreciable influence on the shape of the BSP compared to geometrical depth.

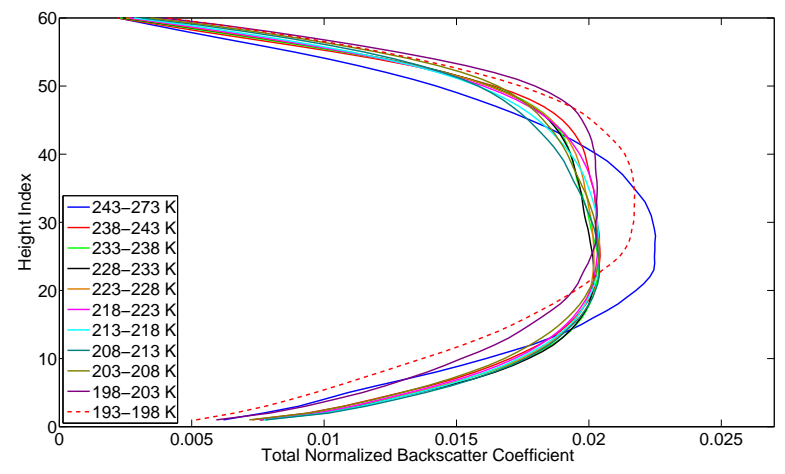

Fig. 9. Mean BSPs for cirrus clouds with selected midlayer temperature intervals. Tropical regions at daytime are considered.

\subsection{Temperature and backscatter profile}

CALIOP Level 2 products contain temperature data derived from the GEOS-5 model and provided by the GMAO Data Assimilation System (http://gmao.gsfc.nasa.gov/index.php) which are exploited in the investigation of the influence of temperature on the shape of the cirrus clouds BSPs. An analysis of the effect of cloud temperature on BSP is performed by using the mid-layer temperature (or midpoint temperature) of the cloud that is defined as the temperature at the geometric midpoint of the layer in the vertical dimension (http: //www-calipso.larc.nasa.gov/resources/calipso_users_guide/ data_summaries/layer/\#layer_top_temperature).

In Fig. 3 it was shown that similar distributions of midlayer temperature characterize diurnal and nocturnal data while some differences arise when latitude is accounted for, due to the higher tropopause at the tropical regions. Since a single temperature value is an acceptable approximation of the cloud temperature state only if geometrically thin clouds are considered, the analysis is limited to those clouds of the FD with vertical extension smaller than $2 \mathrm{~km}$.

In Fig. 9 the annual mean BSPs for cirrus clouds with varying midlayer temperature is shown. Only the tropical case at daytime is considered since results are very similar for both regions and times of the day. Temperature intervals of $5 \mathrm{~K}$ are taken into account from $193 \mathrm{~K}$ to $243 \mathrm{~K}$. Another interval comprises temperatures higher than $243 \mathrm{~K}$. This last interval contains a very limited number of clouds. Results show that mean BSPs are only weakly dependent on temperature. It is observed that, on average, the maximum characterizing the backscatter coefficient normalized curve increases for a decreasing in the mid-layer cloud temperature (the BSP curve becomes more peaked). Some exceptions are anyway present. The effect is observed at both latitudes and at nighttime and daytime. The difference indexes $\left(\mathcal{I}_{\Delta}\right)$ among the BSP curves of same latitude and time of the day (ML day, ML night, tropics day and tropics night) but multiple midpoint temperatures have values that are mostly below $10 \%$. 
The above analysis cannot highlight possible variation in the shape of the BSP as a function of differences in the cloud temperature profile, but it only allows temperature to be considered as a bulk parameter.

In order to tackle possible effects of vertical temperature variations on the shape of BSP an investigation is performed which accounts for clouds with geometrical thickness between 4 and $4.5 \mathrm{~km}$ and analyzes separately cirri with large and small temperature gradients between the cloud top and bottom. For clouds with the above geometrical thickness the top and bottom temperature difference distribution is computed. Mean difference between cloud top and cloud bottom temperature is about 30 and $35 \mathrm{~K}$ respectively for ML and tropics. The cirrus clouds that fall below the first and above the third quartile of the top-bottom temperature difference distribution are compared for both latitudes and for day and night data separately. Results (not shown) do not highlight any significant temperature dependence except for a slight increase, in the daylight case, in the BSP maximum at the cloud base for data with the largest temperature gradient. The difference index computed for BSPs with large and small temperature gradient is anyway very small and less than $8 \%$ for all the situations considered.

It is thus concluded that the influence of temperature on the shape of mean BSPs, both at night and day, is of minor significance if compared to the effects related with the cloud geometrical thickness.

\subsection{Polynomial fitting of mean backscatter profiles}

An analytical representation of mean BSPs measured by CALIOP is given, so that climatological shape of the BSPs of cirrus clouds in different conditions could be used by the reader.

The statistical analysis of BSPs has shown that the cloud geometrical thickness is the main parameter affecting the shape of the mean CALIOP BSP. It is found that a maximum in the mean BSP is placed near the top of the cloud for small $\Delta z$ (less than $2 \mathrm{~km}$ at daytime and $3 \mathrm{~km}$ at nighttime) and moves towards the cloud base with increasing vertical extension. Also the cloud optical depth and temperature (even weakly) affect the shape of the mean CALIOPmeasured BSPs. The main effect of these two quantities is to modify the intensity of the maximum of backscatter (relatively to levels near cloud base or top), which takes the highest values for the highest temperatures and the largest optical depths. Temperature and cloud optical depth produce second-order effects on the shape of backscatter profile and thus only the influence of cloud geometrical thickness is here considered.

Since the shape of BSPs for varying $\Delta z$ shows very limited dependence on latitude (Figs. 5 and 6), only polynomial fittings for the day and night cases are provided.

Mean BSPs obtained for the night and day cases (tropics and midlatitudes are considered together) are fitted with ana- lytical functions. The fitting function used is a two-variables (geometrical depth and height index), fifth-order polynomial:

$\beta(\Delta z, h)=\sum_{j=0}^{4}\left(\Delta z+\frac{p_{0 j}}{p_{1 j}}\right) p_{1 j} h^{j}+p_{05} h^{5}$

where the $h$ variable assumes integer values from 1 to 60 and describes the vertical variation of $\beta$ for a cloud with geometrical thickness equal to $\Delta z$. Thus, given the cloud top altitude $\left(z_{t}\right)$, the quantity derived in Eq. (9) describes a cirrus cloud normalized BS at height $z$. The altitude $z$ is simply derived from:

$z=z_{t}-\Delta z+\frac{h-1}{59} \Delta z$

In Table 5 the polynomial coefficients for the fit of the mean BSPs of night and day cirrus clouds are given. The fit is such that in any case the $\mathcal{I}_{\Delta}$ computed for the analytical and climatological mean BSP curves is less than about $6 \%$.

\section{Application to radiative transfer computations}

\subsection{From mean backscatter profiles to ice-water- content profiles}

One of the important aspects of computing mean BSPs lies in the strict relation between the profile of the backscatter (and extinction) coefficient and the distribution of ice mass along the cloud vertical dimension. The IWC profile largely affects the cloud weighting function and it is thus fundamental for the derivation of the radiative fields and the computation of the energy balance of cloud layers (Maestri and Rizzi, 2003). Hence, climatological information on IWC vertical distribution can potentially improve both the radiative forward models simulations of cloudy fields and inversion algorithms dedicated to the retrieval of cloud properties.

In this regard, the radiative impact of using IWC derived from the mean measured BSPs instead of vertically constant IWC is evaluated for a simple and typical case. Before that it is shown that, under certain conditions, which represent a fairly good approximation of reality, proportionality between the vertical profiles of the backscatter coefficient and the IWC profile exists. If this is the case, the mean BSPs (i.e. the fitting functions described in Eq. 9) can be exploited in forward or inverse radiative transfer models to define the distribution of the ice mass along the cloud vertical dimension thus providing a more realistic description of the cloud structure.

The backscatter coefficient of a collection of ice crystals depends on various parameters such as the total IWC, the particle size distribution, the particle shapes and their orientations. Information on variations of the shape and of the orientation of ice particles along the vertical depth of cirrus clouds is rare. Images, taken from Cloud Particle Imager, of ice particles in regions at the top, middle, and base of clouds 
Table 5. Polynomial coefficients for mean backscatter profiles fitting (Eq. 9).

\begin{tabular}{lcc}
\hline Coeff. & Day & Night \\
\hline$p_{00}$ & $-7.2 \times 10^{-3}$ & $-4.5 \times 10^{-3}$ \\
$p_{01}$ & $2.2 \times 10^{-3}$ & $5.37 \times 10^{-4}$ \\
$p_{02}$ & $-5.46 \times 10^{-5}$ & $5.57 \times 10^{-5}$ \\
$p_{03}$ & $6.37 \times 10^{-7}$ & $-1.69 \times 10^{-6}$ \\
$p_{04}$ & $-2.17 \times 10^{-10}$ & $1.55 \times 10^{-8}$ \\
$p_{05}$ & $-7.98 \times 10^{-11}$ & $-7.86 \times 10^{-11}$ \\
$p_{10}$ & $6.6 \times 10^{-3}$ & $1.5 \times 10^{-3}$ \\
$p_{11}$ & $-2.53 \times 10^{-4}$ & $5.69 \times 10^{-4}$ \\
$p_{12}$ & $-9.16 \times 10^{-6}$ & $-4.17 \times 10^{-5}$ \\
$p_{13}$ & $3.36 \times 10^{-7}$ & $1.04 \times 10^{-6}$ \\
$p_{14}$ & $-2.31 \times 10^{-9}$ & $-6.92 \times 10^{-9}$ \\
\hline
\end{tabular}

have shown the lack of consistency in particle habits as a function of position in cirrus clouds (Lawson et al., 2006). It is thus assumed that the mixture of habits composing the PSD (i.e. the mass percentage amount of crystals in a size bin with a specific shape with respect to the total mass) remains the same along the cloud height and that the crystals are either randomly oriented or horizontally oriented along the full cloud geometrical depth.

Also data regarding the variation of particles' sizes along the vertical of cirrus clouds are few and non-exhaustive (Baran, 2009). Lawson et al. (2006) show that there are only small differences in the average PSDs in the upper portions of cirrus clouds compared to the lower portions, especially for clouds with vertical dimensions larger than $2 \mathrm{~km}$. The main difference consists is a slightly more mass concentration in the lower portion, found mostly in the slightly larger tail of the lower-region average PSD.

Thus, it is similarly assumed that the shape of the PSD (i.e. the effective dimension of the ice mixture) is invariant throughout the cloud vertical extent (variations of the total number of particles and IWC in the vertical are allowed). This assumption is also taken implicitly in the CALIOP retrieval algorithms to derive the extinction coefficient profile, since lidar ratio is taken constant along the cloud geometrical depth.

Currently, the largest part of numerical weather prediction or climate models parameterize (diagnose) the cloud particle dimensions as function of prognostic variables (i.e. temperature and IWC). The same models, subsequently, derive radiative properties from the effective dimensions of ice crystals. According to current particle dimension parameterizations based on in situ collected data (such as Sun and Rikus, 1999; McFarquhar, 2001; Bozzo et al., 2008) the effective dimension of ice crystals within typical cirrus clouds varies less than a factor of three while IWC variations span various orders of magnitude (typically from $10^{-4}$ to $10^{-1} \mathrm{~g} \mathrm{~m}^{-3}$ ).
More importantly, the dependence of BS coefficient due to particle size variation is less than that due to IWC. As an example we have computed (not shown here) the backscatter coefficient of a mixture of particles such as that reported in Bozzo et al. (2008), which is typical of midlatitude cirri. The effective dimensions used span the 16-300 $\mu \mathrm{m}$ range. Results show that the minimum and maximum of the backscatter coefficient are within a factor of four. That means that the effect of particles size fluctuations along the vertical on BS coefficient is of second-order importance with respect to variations in the IWC which, on the other hand, can affect the same radiometric parameter up of several orders of magnitude. This supports the assumption that the shape of the PSD is constant along the cloud thickness in the context of the present research.

For each cloud layer (identified by its height $z_{i}$ ), the particulate backscatter coefficient of an air volume (or volume particulate backscatter coefficient, $\left.\beta\left(z_{i}\right),\left[\left(\mathrm{m}^{-1} \mathrm{sr}^{-1}\right)\right]\right)$ can be written as:

$\beta\left(z_{i}\right)=\int_{r_{\min }}^{r_{\max }} P(\pi, r) \cdot \sigma_{s}(r) \cdot n\left(r, z_{i}\right) d r$

where $P(\pi, r)$ is the value of the phase function of the particle with effective dimension $r$ at a scattering angle of 180 degrees, $\sigma_{s}(r)$ is the particle scattering cross section $\left[\mathrm{m}^{2}\right]$ and $n\left(r, z_{i}\right)$ is the particle size distribution $\left[\# /\left(\mathrm{m}^{3} \mathrm{~m}\right)\right]$ at level $z_{i}$ with a total particle number concentration equal to $N\left(z_{i}\right)$ $\left[\# / \mathrm{m}^{3}\right]$.

Considering the scattering cross section per unit mass (i.e., the particle-mass-scattering cross section $\left.k_{\mathrm{S}}(r)\left[\mathrm{m}^{2} \mathrm{~kg}^{-1}\right]\right)$ a mean mass-scattering cross section can be defined as:

$\overline{k_{\mathrm{s}}}=\frac{\int k_{\mathrm{s}}(r) n\left(r, z_{i}\right) d r}{\int n\left(r, z_{i}\right) d r}$

where the integration is performed over all the particles effective dimensions. Under the assumption of having the same PSD shape in all the cloud layers (i.e. $n\left(r, z_{i}\right) / N\left(z_{i}\right)$ does not depend on $z_{i}$ ), the mean mass scattering cross section becomes a layer independent quantity. For the same conditions, it is possible to define a mean phase function at the backscattering angle:

$$
\begin{aligned}
\overline{P(\pi)} & =\frac{\int P(\pi, r) \cdot k_{\mathrm{S}}(r) \cdot n\left(r, z_{i}\right) d r}{\int k_{\mathrm{S}}(r) \cdot n\left(r, z_{i}\right) d r} \\
& =\frac{\int P(\pi, r) \cdot k_{\mathrm{S}}(r) \cdot \frac{n\left(r, z_{i}\right)}{N\left(z_{i}\right)} d r}{\bar{k}_{\mathrm{S}}}
\end{aligned}
$$

which is also a layer independent quantity. Similarly, a mean scattering cross section is defined as:

$$
\overline{\sigma_{s}}=\frac{\int \sigma_{s}(r) n\left(r, z_{i}\right) d r}{\int n\left(r, z_{i}\right) d r}
$$


Thus the (volume) backscatter coefficient is re-written in terms of mean quantities:

$\beta\left(z_{i}\right)=\overline{P(\pi)} \cdot \overline{\sigma_{s}} \cdot N\left(z_{i}\right)$

Since a simple relation between the mean scattering cross section $\left(\overline{\sigma_{s}}\right)$ and the total ice-water-content of the $i$-th layer (i.e., $\left.\operatorname{IWC}\left(z_{i}\right)\left[\mathrm{kg} \mathrm{m}^{-3}\right]\right)$ exists:

$\overline{\sigma_{s}}=\frac{\overline{k_{\mathrm{s}}} \cdot \operatorname{IWC}\left(z_{i}\right)}{N\left(z_{i}\right)}$

the volume backscatter coefficient of the $i$-th layer is written as:

$\beta\left(z_{i}\right)=\overline{P(\pi)} \cdot \overline{k_{\mathrm{s}}} \cdot \operatorname{IWC}\left(z_{i}\right)$

At visible wavelengths, the single scattering albedo of a distribution of pristine ice crystals is equal to one and the massextinction cross section $k_{\mathrm{e}}(r)$ can be used in place of $k_{\mathrm{s}}(r)$. The volume backscatter coefficient is then:

$\beta\left(z_{i}\right)=\overline{P(\pi)} \cdot \overline{k_{\mathrm{e}}} \cdot \operatorname{IWC}\left(z_{i}\right)$

The cloud total optical thickness, $\tau$, as a function of the icewater-content is:

$\tau=\sum_{i=1}^{n} k_{\mathrm{e}}\left(z_{i}\right) \cdot \operatorname{IWC}\left(z_{i}\right) \cdot \delta z_{i}$

where $\delta z_{i}$ is the geometrical depth of the $i$-th cloud layer.

Using the definition of Ice-Water-Path (namely the ice content in the whole cloud depth, $\left[\mathrm{kg} \mathrm{m}^{-2}\right]$ ):

$\operatorname{IWP}=\sum_{i=1}^{n} \operatorname{IWC}\left(z_{i}\right) \cdot \delta z_{i}$

and recalling the assumption of having the same PSD shape at each cloud layer, the optical thickness is:

$\tau=\overline{k_{\mathrm{e}}} \cdot \sum_{i=1}^{n} \operatorname{IWC}\left(z_{i}\right) \cdot \delta z_{i}=\overline{k_{\mathrm{e}}} \cdot \operatorname{IWP}$

Using Eq. (18) and Eq. (21) it is easily found that:

$\operatorname{IWC}\left(z_{i}\right)=\frac{\operatorname{IWP}}{\tau \cdot \overline{P(\pi)}} \cdot \beta\left(z_{i}\right)$

Equation (22) highlights that a (linear) proportionality exists between the backscatter coefficient and the IWC when the PSD shape (and same particle mixture of habits and orientation) is assumed constant along the cloud vertical extent. The constant of proportionality depends only on total ice content and optical thickness (bulk properties) and on the mean phase function at the backscattering angle. Thus, climatological IWC profiles can be derived by exploiting the polynomial functions given in the previous section.

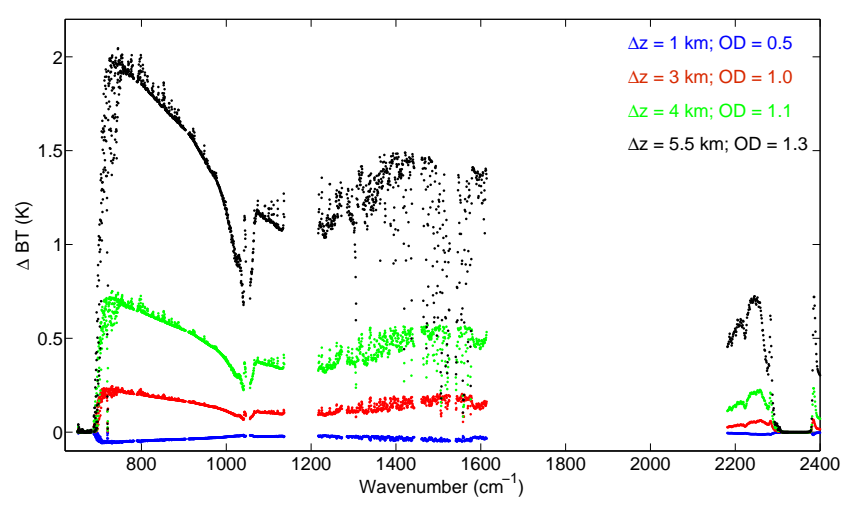

Fig. 10. AIRS brightness temperature differences for pairs of clouds with same OD but IWC profile that in one case is derived from climatological BSPs and in the other case is assumed constant along the cloud vertical. Four different geometrical thicknesses are considered in the example. Typical ODs are associated to each thickness.

\subsection{Radiative impact}

The radiative impact of using CALIOP-derived IWC profiles instead of homogeneous ones for cirrus clouds is evaluated. A realistic atmospheric column in presence of a cirrus cloud is chosen for the simulations. A cirrus case detected on July 2008 in the Atlantic Ocean, at about $1000 \mathrm{~km}$ south-west off the coast of Sierra Leone $\left(1.40^{\circ} \mathrm{N}, 19.11^{\circ} \mathrm{W}\right)$ is selected. In the simulations cloud top is fixed at an altitude of $13.5 \mathrm{~km}$ (as the measured one), while cloud thickness is varied from 1 to $5.5 \mathrm{~km}$. The cloud OD is determined from Eq. (7) so that typical conditions are reproduced (see green curve in Fig. 7).

For every $\Delta z$ value two simulations are performed: in one case the IWC profile is derived from the fitting functions of daylight BSPs described in subsection 3.6 (IWC-cal), in the other case it is assumed constant along the cloud vertical extent (IWC-const). The brightness temperature (BT) differences between the two assumptions and for the four geometrical thicknesses are shown in Fig. 10. The simulated spectral radiances from which the BTs are derived are computed by using the multiple scattering line-by-line radiative transfer code described by Rizzi et al. (2007) and Maestri et al. (2010).

Simulations results are weighted by the spectral response functions of the Atmospheric InfraRed Sounder (AIRS) so that a realistic measurement situation is reproduced. The AIRS sensor is chosen so that the impact of the assumptions concerning the IWC profile on radiometric signals could be evaluated for high spectral resolution key-bands in the infrared spectrum. AIRS is carried by the Aqua satellite, it flies on the same A-Train formation with CALIPSO (about $75 \mathrm{~s}$ before CALIPSO) and, in view of a combined use of AIRS and CALIOP data, a relatively simple data collocation is possible. The assumed cloud particle mixture is described 
by Bozzo et al. (2008) and has been already used by Maestri et al. (2010). Particles effective dimension is assumed to be $40 \mu \mathrm{m}$. Information about temperature and humidity profiles are derived directly from the CPro file. Climatological values of the main atmospheric gases are also used.

The simulations in Fig. 10 show a noteworthy impact of the spectral BTs to assumptions on IWC profiles. For the smaller vertical dimension ( $\Delta z$ equal to $1 \mathrm{~km}$ ), given the simulation conditions of the example (typical of tropical cirri), the BTs derived from IWC-cal minus those derived from IWC-const show small negative values. The result is a consequence of the highly peaked distribution of IWC close to cloud top in case of IWC-cal that implies lower cloud emitting temperatures (for a typical temperature profile). For $\Delta z$ larger than $3 \mathrm{~km}$ the IWC profiles derived from the climatological BSPs provide weighting functions with larger values at lower cloud altitudes and thus higher emitting temperatures. The residuals between the two sets of simulations become larger as the geometrical depth increases as a consequence of the growth of the maximum of the BSP near cloud base. The differences in BT are both dependent on geometrical thickness and optical depth values and in the example only typical combinations of these two parameters are reported.

From the Fig. 10 it is derived that, given the high altitude of the cloud case considered (and of all the clouds analyzed in this work), significant BT differences due to the assumptions on IWC profile are observed not only in the main atmospheric windows $\left(750-1100\right.$ and $\left.1180-1250 \mathrm{~cm}^{-1}\right)$, but also in those spectral channels whose gaseous weighting functions peak in the medium-lower troposphere (i.e. for the AIRS water-vapor channels between 1250 and $1613 \mathrm{~cm}^{-1}$ ). This demonstrates that, cirrus cloud IWC profiles have important impacts on all the high-resolution radiances between 300 and $2400 \mathrm{~cm}^{-1}$ except for the most absorbing $\mathrm{CO}_{2}$ (around 667 and $2300 \mathrm{~cm}^{-1}$ ) and far-infrared water-vapor channels. Note that AIRS noise-equivalent-temperature difference is $0.07-0.40 \mathrm{~K}$ in the spectral interval $3.75-11 \mu \mathrm{m}$ (i.e. from 909 to $2666 \mathrm{~cm}^{-1}$ ) at $250 \mathrm{~K}$.

\section{Summary}

Vertical variations of cloud features (especially mass) sensibly impact on the clouds and atmospheric energy balance. In particular, cirrus cloud properties along the vertical dimension are poorly known and a better representation of their physical and optical characteristics will be advantageous both for (among others) the derivation of cloud products by inverse algorithms and for forward modeling radiative transfer computations.

The present work aims at contributing to a better knowledge and understanding of the characterization of the ice cloud properties along their vertical extent. More specifically, the goal of this paper is to provide a statistical anal- ysis of satellite measured backscatter and extinction profile in presence of cirrus clouds. A detailed examination of the shape of vertical backscatter profile (BSP) of ice clouds in the upper troposphere of tropics and midlatitudes is then performed.

Measurements collected by the CALIOP lidar on-board of the Cloud-Aerosol Lidar and Infrared Pathfinder Satellite Observations (CALIPSO) during the year 2008 over the oceans are considered. A data set is built containing clouds with maximum optical depth of 2 , so that the full cloud vertical extent is characterized by CALIOP measures and possible total attenuation effects on the backscatter signal are avoided. Cirrus cloud covered fields of view are selected even if multilayered clouds are detected, provided that the cirrus is the highest cloud sensed by CALIOP. The number of cases accounted for covers more than $33 \%$ of all the CALIOP fields of view (with spatial resolution equals to $5 \mathrm{~km}$ ) from $60^{\circ} \mathrm{S}$ to $60^{\circ} \mathrm{N}$, denoting a widespread presence of high, thin, iced layers over large part of the ocean's globe.

Among these cases, only those with nearly homogeneous cloud top and base altitude (derived from an analysis at the higher spatial resolution of $1 \mathrm{~km}$ ) are considered for the investigation of the shape of CALIOP backscatter and extinction profiles. The selection of horizontally homogeneous cirrus clouds only is performed to avoid possible artifacts in the definition of climatological BSPs. Homogeneous cirri are present in the 6-9\% of the CALIOP 5-km fields of view that is about one-fourth of all the detected cirrus clouds. For the first time, distributions of physical features of homogeneous cirrus clouds are provided for tropics and midlatitudes and daytime and nighttime and compared to the whole ensemble of cirri.

The selected BSPs are first vertically rescaled to a standard length and to a specific number of points and then their areas (from cloud-base to cloud-top altitude) are normalized. In this way, a comparison among the shapes of BSPs that differ in the number of scattering layers and in the total amount of energy backscattered to the lidar is made possible.

Midlatitude and tropical data, such as daylight and nighttime measurements, are analyzed separately due to distinctive mean cloud physical characteristics. In particular, it is observed that horizontally homogeneous tropical cirri have higher mean top height and midpoint temperature, and lower optical depth with respect to midlatitude ones. Distributions of geometrical thickness are very similar at the two latitudes, but differ from day and night showing frequently larger depths for the nighttime case.

Annual means of BSPs are very similar for tropics and ML while noticeable differences are found when daylight and nighttime data are compared. The monthly averages of the shape of the BSPs are all very similar and the dependence on the time of the year is then neglected.

The relationship between the shape of the measured BSPs and cloud and environmental physical parameters (such as cloud optical depth, geometrical thickness and temperature) 
is investigated. Possible influences of the instrument and retrieval algorithms on the shape of the BSPs (i.e., possible attenuation correction errors in deriving backscattering and extinction coefficients) are not accounted for, but the reader should be aware of this possibility.

It is found that the cloud geometrical thickness is the main parameter affecting the shape of the mean CALIOP BSP. Specifically, cirrus clouds with thin geometrical thicknesses (less than 2 or $3 \mathrm{~km}$ depending on the period of the day) show that a maximum in the normalized BS profile is placed near cloud top. As the cloud geometrical thickness increases the maximum of the mean BSP curve is shifted towards the cloud base. Nighttime and daytime data show some differences even when cirrus clouds within the same thickness are compared, suggesting that possibly different physical features characterizing the two cases might influence the shape of the BSP.

Similarly to what was found for the geometrical thickness also the optical depth analysis shows that the maximum in the shape of the mean BS curves is close to the cloud top for clouds with very thin optical depth and to the base for the optically thickest clouds of the database. It is observed that a monotonic geometrical thickness - optical depth dependence characterizes the ensemble of cirrus clouds under study. In this regard, a parameterization that derives mean optical depth (and its standard deviation) as function of the cloud geometrical thickness is provided.

To minimize the cloud geometrical thickness - optical depth dependence, mean BSPs are computed for cirrus clouds with nearly constant geometrical thickness and multiple optical depth intervals. Results show limited variability of the mean BSPs compared to that found when mean BSPs of cirrus clouds with constant OD and varying geometrical thickness are computed.

The effects of cloud temperature and of temperature gradients within the cloud on the shape of the BSPs are also investigated. Results show a smaller influence of the temperature parameter with respect to the geometrical thickness.

It is observed that the BSP maximum slightly increases as the cloud temperature decreases and as the optical depth increases.

Analytical functions are provided to fit the mean BSPs so that climatological backscatter profiles can be easily used in forward or inverse models. It is, in fact, demonstrated that under certain (realistic) assumptions, which are consistent with assumptions made in the CALIOP retrieval algorithm, the mean backscatter (and extinction) profile is linearly proportional to the cloud IWC profile. Since temperature and cloud optical depth produce second-order effects on the shape of backscatter profile and since the shape of BSPs for varying geometrical thickness shows very limited dependence on latitude, the BSP parameterization in terms of varying geometrical thickness is given for day and night data only.
The fitting polynomial functions are applied in radiative transfer simulations of the Atmospheric Infrared Sounder (AIRS) measured radiances. The impact, on infrared brightness temperatures, of using either a constant ice-watercontent (IWC) in the cloud vertical dimension or an IWC profile derived from the BSP fitting functions is evaluated for a typical case of tropical cirrus over the ocean. Results show that, for multiple combinations of geometrical thickness and optical depth, brightness temperature differences in two cases are above the AIRS noise equivalent temperature difference.

\section{Appendix A}

\section{Definition of the minimum number of elements defining the data subset}

In Sect. 3.2 it is shown that the variability among measured profiles can be very large. Nevertheless, averages performed over sufficiently large subsets (i.e. over one month of data or one season) converge to well defined mean curves.

It is our goal to find how many profiles $(N)$ are needed to obtain a mean profile which is representative of an ideally infinite set of data of the same type. The problem arises when the full data set under study (the FD) is divided into smaller subsets and statistical analysis are performed on a limited number of profiles. The question that we are trying to answer in this Appendix is the following: is the subset well representative of an ideally infinite number of backscatter profiles of cirrus clouds with similar characteristics?

The minimum number of profiles, $N$, which is accepted in order to apply a statistical analysis, is computed with the following methodology. Given the full data set of cirrus BS profiles, the number of elements required to obtain a mean BSP "sufficiently close" to the one derived from the entire data set is evaluated. A sufficient closeness among the means (of the subset and of the full extended database) is defined through the $\mathcal{I}_{\Delta}$ which must be less than $1 \%$.

The mean of the subset is computed as:

$\left\langle\beta_{s}\left(z_{i}\right)\right\rangle=\frac{1}{N} \sum_{j=1}^{N} \beta_{j}\left(z_{i}\right)$

where the $N$ BSPs are selected randomly within the whole data set. The value found in equation A1 is computed $M$ different times $(M=1000$ in our case), i.e. for $M$ (1000) distinct subsets of $N$ randomly selected profiles. Each time the mean of the subset is compared to the mean of the entire data set and an $\mathcal{I}_{\Delta}$ is computed. Then the average of the difference index is computed:

$\left\langle\mathcal{I}_{\Delta}\right\rangle=\frac{1}{M} \sum_{m=1}^{M} \mathcal{I}_{\Delta, m}$

The entire procedure is iterated. $N$ is increased at each step until the value of $\left\langle\mathcal{I}_{\Delta}\right\rangle$ is less than $1 \%$. 
This methodology, applied to the cirrus cloud data set used in this work, provides a value for $N$ that is of the order of 1500. This will be the minimum number of profiles in our subsets that is required to perform averaging operations.

\begin{tabular}{ll}
\hline \multicolumn{2}{l}{ List of acronyms } \\
\hline AIRS & Atmospheric Infrared Sounder \\
BS & Backscatter \\
BSP & Backscatter profile \\
BT & Brightness temperature \\
CAD & Cloud-Aerosol Discrimination \\
CALIOP & Cloud-Aerosol Lidar with Orthogo- \\
& nal Polarization \\
CALIPSO & Cloud-Aerosol Lidar and Infrared \\
& Pathfinder \\
& Satellite Observation \\
CLay & Cloud layer \\
CM & Climate model \\
CPro & Cloud profile \\
CPR & Cloud Profiling Radar \\
ETP & Extinction profile \\
FD & Final database \\
FOV & Field of view \\
GEOS & Goddard Earth Observation System \\
GLAS & Geoscience Laser Altimeter System \\
GMAO & Global Modeling Assimilation Office \\
iFOV & Instantaneous field of view \\
IWC & Ice water content \\
IWP & Ice water path \\
LITE & Lidar In-space Technology \\
ML & Experiment \\
MODIS & Midlatitudes \\
& Moderate Resolution Imaging \\
NWP & Spectroradiometer \\
OD & Numerical weather prediction \\
PSD & Optical depth \\
RD & Particle size distribution \\
SNR & Reduced database \\
TRO & Signal-to-noise ratio \\
UTC & Tropics \\
& Coordinated Universal Time \\
& \\
& \\
&
\end{tabular}

List of symbols

\begin{tabular}{|c|c|c|}
\hline Symbol & Units & Description \\
\hline$\beta$ & {$\left[\mathrm{m}^{-1} \mathrm{sr}^{-1}\right]$} & $\begin{array}{l}\text { Total particulate backscatter } \\
\text { coefficient }\end{array}$ \\
\hline$\beta^{\prime}$ & {$\left[\mathrm{m}^{-1} \mathrm{sr}^{-1}\right]$} & $\begin{array}{l}\text { Total particulate and molecular } \\
\text { backscatter coefficient }\end{array}$ \\
\hline$\beta_{a}$ & {$\left[\mathrm{~m}^{-1} \mathrm{~s}\right.$} & $\begin{array}{l}\text { Attenuated particulate and } \\
\text { molecular backscatter } \\
\text { coefficient }\end{array}$ \\
\hline
\end{tabular}

\begin{tabular}{|c|c|c|}
\hline$\beta_{n}$ & & $\begin{array}{l}\text { Total normalized particulate } \\
\text { backscatter coefficient }\end{array}$ \\
\hline$\Delta z$ & {$[\mathrm{~m}]$} & Cloud geometrical thickness \\
\hline$\delta z_{i}$ & {$[\mathrm{~m}]$} & $\begin{array}{l}\text { Geometrical depth of the } i \text {-th } \\
\text { layer of the cloud }\end{array}$ \\
\hline$h$ & & Height index \\
\hline $\mathcal{I}_{\Delta}$ & {$[\%]$} & Difference index \\
\hline IWC & {$\left[\mathrm{kg} \mathrm{m}^{-3}\right]$} & Ice Water Content \\
\hline IWP & {$\left[\mathrm{kg} \mathrm{m}^{-2}\right]$} & Ice Water Path \\
\hline$k_{\mathrm{e}}(r)$ & {$\left[\mathrm{m}^{2} \mathrm{~kg}^{-1}\right]$} & Mass-extinction cross section \\
\hline$k_{\mathrm{S}}(r)$ & {$\left[\mathrm{m}^{2} \mathrm{~kg}^{-1}\right]$} & Mass-scattering cross section \\
\hline$n(r)$ & {$\left[\# /\left(\mathrm{m}^{3} \mathrm{~m}\right)\right]$} & Particle size distribution \\
\hline$P(\pi, r)$ & {$\left[\mathrm{sr}^{-1}\right]$} & $\begin{array}{l}\text { Phase function for dimension } \\
\text { and scattering angle of } 180 \\
\text { degrees }\end{array}$ \\
\hline$S_{P}$ & {$\left[\mathrm{sr}^{-1}\right]$} & Particulate lidar ratio \\
\hline . & {$\left[\mathrm{m}^{-1}\right]$} & Particulate extinction coefficient \\
\hline$\sigma_{s}$ & {$\left[\mathrm{~m}^{2}\right]$} & Particle scattering cross section \\
\hline$\sigma_{\tau}$ & & $\begin{array}{l}\text { Mean optical depth standard } \\
\text { deviation }\end{array}$ \\
\hline $\mathcal{T}^{2}$ & & Two-way transmittance \\
\hline 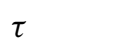 & & Cloud optical depth, OD \\
\hline $\mathcal{Z} b, 2$ & & $\begin{array}{l}\text { Thresholds for cloud top and base } \\
\text { used in the homogenization } \\
\text { process }\end{array}$ \\
\hline & {$[\mathrm{m}$} & Altitude \\
\hline & {$[\mathrm{m}]$} & Cloud top and base altitude \\
\hline
\end{tabular}

Acknowledgements. The data used in this work were obtained from the NASA Langley Atmospheric Science Data Center to which goes our gratitude. The authors also wish to thank R. Rizzi of the University of Bologna and R. Holz of the University of Wisconsin-Madison for their valuable suggestions.

Edited by: B. Mayer

\section{References}

Anselmo, T., Clifton, R., Hunt, W., Lee, K.-P., Murray, T., Powell, K., Rodier, S. D., Vaughan, M., Chomette, O., Viollier, M., Hagolle, O., Lifermann, A., Garnier, A., and Pelon, J.: Data Management System, Data Product Catalog, 2007.

Baran, A. J.: A review of the light scattering properties of cirrus, J. Quant. Spectrosc. Ra., 110, 1239-1260, doi:10.1016/j.jqsrt.2009.02.026, 2009.

Baum, B. A., Heymsfield, A. J., Yang, P., and Bedka, S. T.: Bulk scattering properties for the remote sensing of ice clouds. Part I: Microphysical data and models, J. Appl. Meteorol., 44, 18851895, 2005a.

Baum, B. A., Yang, P., Heymsfield, A. J., Platnick, S., King, M. D., Hu, Y., and Bedka, S. T.: Bulk scattering properties for the remote sensing of ice clouds. Part II: Narrowband Models, J. Appl. Meteorol., 44, 1896-1911, 2005b.

Berthier, S., Chazette, P., Pelon, J., and Baum, B.: Comparison of cloud statistics from spaceborne lidar systems, Atmos. Chem. Phys., 8, 6965-6977, doi:10.5194/acp-8-6965-2008, 2008. 
Bozzo, A., Maestri, T., Rizzi, R., and Tosi, E.: Parameterization of single scattering properties of mid-latitude cirrus clouds for fast radiative transfer models using particle mixtures, Geophys. Res. Lett., 35, L16809, doi:10.1029/2008GL034695, 2008.

Chiriaco, M., Chepfer, H., Noel, V., Haeffelin, M., and Drobinski, P.: Dual lidar observations at $10.6 \mu \mathrm{m}$ and $532 \mathrm{~nm}$ for retrieving semitransparent cirrus cloud properties, J. Appl. Meteorol., 45, 537-555, doi:10.1175/JAM2435.1, 2006.

Cho, H.-M., Yang, P., Kattawar, G. W., Nasiri, S. L., Hu, Y., Minnis, P., Trepte, C., and Winker, D.: Depolarization ratio and attenuated backscatter for nine cloud types: analyses based on collocated CALIPSO lidar and MODIS measurements, Opt. Express, 16, 3931-3948, doi:10.1364/OE.16.003931, 2008.

Eguchi, N., Yokota, T., and Inoue, G.: Characteristic of cirrus clouds from ICESat/GLAS observations, Geophys. Res. Lett., 34, L09810, doi:10.1029/2007GL029529, 2007.

Heymsfield, A. J. and Miloshevich, L. M.: Relative Humidity and temperature influences on cirrus formation and evolution: observations from wave clouds and FIRE II, J. Atmos. Sci., 52, 43024326, 1995.

Heymsfield, A. J. and Miloshevich, L. M.: Parameterizations for the cross-sectional area and extinction of cirrus and stratiform ice cloud particles, J. Atmos. Sci., 60, 936-956, 2003.

Holton, J. R.: An introduction to dynamic meteorology, vol. 88 of International Geophyisics Series, Elsevier Academic Press, Burlington, MA, USA, 4th edn., 2004.

Holz, R. E., Ackerman, S. A., Nagle, F. W., Frey, R., Dutcher, S., Kuehn, R. E., Vaughan, M. A., and Baum, B.: Global Moderate Resolution Imaging Spectroradiometer (MODIS) cloud detection and height evaluation using CALIOP, J. Geophys. Res., 113, D00A19, doi:10.1029/2008JD009837, 2008.

King, M. D., Platnick, S., Yang, P., Arnold, G. T., Gray, M. A., Riedi, J. C., Ackerman, S. A., and Liou, K. N.: Remote sensing of liquid water and ice cloud optical thickness and effective radius in the arctic: Application of airborne multispectral MAS data, J. Atmos. Ocean. Tech., 21, 857-875, 2004.

Lawson, R. P., Baker, B., Pilson, B., and Mo, Q.: In situ observations of the microphysical properties of wave, cirrus, and anvil clouds. Part II: cirrus clouds, J. Atmos. Sci., 63, 3186-3203, 2006.

Liou, K. N.: Influence of cirrus clouds on weather and climate processes: a global perspective, Mon. Weather Rev., 114, 11671199, 1986.

Lynch, D. K.: Cirrus, Oxford University Press, Inc., New York, NY, USA, 2002.

Maestri, T. and Holz, R. E.: Retrieval of cloud optical properties from multiple infrared hyperspectral measurements: A methodology based on a line-by-line multiplescattering code, IEEE T. Geosci. Remote, 47, 2413-2426, doi:10.1109/TGRS.2009.2016105, 2009.

Maestri, T. and Rizzi, R.: A study of infrared diabatic forcing of ice clouds in the tropical atmosphere, J. Geophys. Res., 108, 4139, doi:10.1029/2002JD002146, 2003.

Maestri, T., Rizzi, R., and Smith, J. A.: Spectral infrared analysis of a cirrus cloud based on Airborne Research Interferometer Evaluation System (ARIES) measurements, J. Geophys. Res., 110, D06111, doi:10.1029/2004JD005098, 2005.
Maestri, T., Girolamo, P. D., Summa, D., and Rizzi, R.: Clear and cloud sky investigations using Raman lidar and airborne interferometric measures from the European AQUA Thermodynamic Experiment, Atmos. Res., 97, 157-169, doi:10.1016/j.atmosres.2010.03.020, 2010.

Martins, E., Noel, V., and Chepfer, H.: Properties of cirrus and subvisible cirrus from nighttime Cloud-Aerosol Lidar with Orthogonal Polarization (CALIOP), related to atmospheric dynamics and water vapor, J. Geophys. Res., 116, D02208, doi:10.1029/2010JD014519, 2011.

McFarquhar, G. M.: Comments on "Parameterization of effective sizes of cirrus-cloud particles and its verification against observations" by Zhian Sun and Lawrie Rikus. October B, 1999, 125, 3037-3055, 1999, 125, 3037-3055, Q. J. Roy. Meteor. Soc., 127, 261-265, doi:10.1002/qj.49712757115, 2001.

McFarquhar, G. M. and Heymsfield, A. J.: Microphysical characteristic of three anvils sampled during the central equatorial pacific experiment, J. Atmos. Sci., 53, 2401-2423, 1996.

McFarquhar, G. M., Heymsfield, A. J., Spinhirne, J., and Hart, B.: Thin and subvisual tropopause tropical cirrus: observations and radiative impacts, J. Atmos. Sci., 57, 1841-1853, 2000.

Nazaryan, H., McCormick, M. P., and Menzel, W. P.: Global characterization of cirrus clouds using CALIPSO data, J. Geophys. Res., 113, D16211, doi:10.1029/2007JD009481, 2008.

Rizzi, R., Maestri, T., and Bozzo, A.: Reference tool for simulation of VIS/NIR spectra radiances, ADGB, Dipartimento di Fisica, Universitá di Bologna, 2007.

Sassen, K., Wang, Z., and Liu, D.: Global distribution of cirrus clouds from CloudSat/Cloud-Aerosol Lidar and Infrared Pathfinder Satellite Observations (CALIPSO) measurements, J. Geophys. Res., 113, D00A12, doi:10.1029/2008JD009972, 2008.

Sun, Z. and Rikus, L.: Parameterization of effective sizes of cirrus-cloud particles and its verification against observations, Q. J. Roy. Meteor. Soc., 125, 3037-3055, doi:10.1002/qj.49712556012, 1999.

Wang, X., Liou, K. N., Ou, S. S. C., Mace, G. G., and Deng, M.: Remote sensing of cirrus cloud vertical size profile using MODIS data, J. Geophys. Res., 114, doi:10.1029/2008JD011327, 2009.

Wendisch, M., Pilewskie, P., Pommier, J., Howard, S., Yang, P., Heymsfield, A. J., Schmitt, C. G., Baumgardner, D., and Mayer, B.: Impact of cirrus crystal shape on solar spectral irradiance: A case study for subtropical cirrus, J. Geophys. Res., 110, D03202, doi:10.1029/2004JD005294, 2005.

Wylie, D. P., Menzel, W. P., Woolf, H. M., and Strabala, K. I.: Four years of global cirrus cloud statistics using HIRS, J. Climate, 7, 1972-1986, 1994.

Wylie, D. P., Jackson, D. L., Menzel, W. P., and Bates, J. J.: Trends in global cloud cover in two decades of HIRS observations, J. Climate, 18, 3021-3031, 2005.

Zhang, Z., Platnick, S., Yang, P., Heidinger, A. K., and Comstock, J. M.: Effects of ice particle size vertical inhomogeneity on the passive remote sensing of ice clouds, J. Geophys. Res., 115, D17203, doi:10.1029/2010JD013835, 2010. 